\title{
Gadgets and Anti-Gadgets Leading to a Complexity Dichotomy
}

\author{
JIN-YI CAI, University of Wisconsin-Madison, USA \\ MICHAEL KOWALCZYK, Northern Michigan University, USA \\ TYSON WILLIAMS, Blocher Consulting, USA
}

We introduce an idea called anti-gadgets for reductions in complexity theory. These anti-gadgets are presented as graph fragments, but their effect is equivalent to erasing the presence of other graph fragments, as if we had managed to include a negative copy of a certain graph gadget. We use this idea to prove a complexity dichotomy theorem for the partition function $Z(G)$ of spin systems over 3-regular directed graphs $G$,

$$
Z(G)=\sum_{\sigma: V(G) \rightarrow\{0,1\}} \prod_{(u, v) \in E(G)} f(\sigma(u), \sigma(v)),
$$

where each edge is given a (not necessarily symmetric) complex-valued binary function $f:\{0,1\}^{2} \rightarrow \mathbb{C}$. We show that $Z(G)$ is either computable in polynomial time or \#P-hard, depending explicitly on $f$. When the input graph $G$ is planar, there is an additional class of polynomial time computable partition functions $Z(G)$, while everything else remains \#P-hard. Furthermore, this additional class is precisely those that can be transformed by a holographic reduction to matchgates, followed by the Fisher-Kasteleyn-Temperley algorithm via Pfaffians.

CCS Concepts: • Theory of computation $\rightarrow$ Problems, reductions and completeness; Constraint and logic programming; • Mathematics of computing $\rightarrow$ Combinatorial algorithms;

Additional Key Words and Phrases: Holant problem, partition function, dichotomy theorem, holographic algorithms, interpolation, counting complexity

\section{ACM Reference format:}

Jin-Yi Cai, Michael Kowalczyk, and Tyson Williams. 2019. Gadgets and Anti-Gadgets Leading to a Complexity Dichotomy. ACM Trans. Comput. Theory 11, 2, Article 7 (February 2019), 26 pages.

https://doi.org/10.1145/3305272

\section{INTRODUCTION}

Reduction, the method of transforming one problem to another and thereby proving the hardness of a problem for an entire complexity class, is arguably the most successful tool in complexity theory to date. When expressed in terms of graph problems, a typical reduction from a problem $\Pi_{1}$

A preliminary version of this article appeared in Innovations in Theoretical Computer Science 2012 [15]. This work is supported by NSF grant nos. CCF-1217549 and CCF-1714275.

Authors' addresses: J.-Y. Cai, University of Wisconsin-Madison, Computer Sciences, Madison, WI, USA; email: jyc@cs.wisc.edu; M. Kowalczyk, Northern Michigan University, Mathematics and Computer Science, Marquette, MI, USA; email: mkowalcz@nmu.edu; T. Williams, Blocher Consulting, Champaign, IL, USA; email: tdw@cs.wisc.edu.

Permission to make digital or hard copies of all or part of this work for personal or classroom use is granted without fee provided that copies are not made or distributed for profit or commercial advantage and that copies bear this notice and the full citation on the first page. Copyrights for components of this work owned by others than the author(s) must be honored. Abstracting with credit is permitted. To copy otherwise, or republish, to post on servers or to redistribute to lists, requires prior specific permission and/or a fee. Request permissions from permissions@acm.org.

(C) 2019 Copyright held by the owner/author(s). Publication rights licensed to ACM.

1942-3454/2019/02-ART7 \$15.00

https://doi.org/10.1145/3305272 
to another problem $\Pi_{2}$ is carried out by designing a gadget-a graph fragment with some desirable properties. The reduction starts from an instance graph $G_{1}$ for $\Pi_{1}$ and introduces one or more copies of the gadget to obtain an instance graph (or possibly multiple instance graphs) $G_{2}$ for $\Pi_{2}$.

The graph $G_{2}$ may contain a polynomial number of copies of the gadget. But can it include some negative copies of a gadget? Of course not-the notion of a negative graph fragment seems meaningless. However, in this article, we introduce an idea for designing reductions that has the effect of introducing negative copies of a gadget in a complexity reduction. More precisely, we show that our new construction idea, when expressed in algebraic terms, has the same effect as erasing the presence of a graph fragment. It is as if we managed to include a negative copy of a certain gadget. We call this an anti-gadget. We think of it as analogous to the pairing of a particle and its anti-particle in physics. We demonstrate the elegance and usefulness of anti-gadgets by proving a new complexity dichotomy theorem in counting complexity where anti-gadgets play a decisive role. Furthermore, we show that anti-gadgets provide a simple explanation for some miraculous cancellations that were observed in previous results $[13,14]$. We also observe how anti-gadgets can guide the search for such gadget sets with cancellations, more by design than by chance.

The new dichotomy theorem that we prove using anti-gadgets can be stated in terms of spin systems over 3-regular graphs with vertices taking values in $\{0,1\}$ and an arbitrary complex-valued ${ }^{1}$ edge function $f(\cdot, \cdot)$ that is not necessarily symmetric.

Define the partition function on $G=(V, E)$ as

$$
Z(G)=\sum_{\sigma: V \rightarrow\{0,1\}} \prod_{(u, v) \in E} f(\sigma(u), \sigma(v)) .
$$

Depending on the nature of the edge function $f$, we show that the problem $Z(\cdot)$ is either computable in $\mathrm{P}$ (called tractable), or \#P-hard. More precisely, the problem is \#P-hard unless the edge function is ( $i$ ) degenerate, (ii) generalized equality, (iii) generalized disequality, or is (iv) affine after a holographic transformation. For these four classes of functions, the problem is computable in polynomial time. Furthermore, if the input is restricted to planar graphs, then the class of tractable problems is augmented by those that are solvable by holographic algorithms with matchgatesall other problems remain \#P-hard. Thus, holographic algorithms with matchgates are a universal methodology for this class of counting problems $Z(G)$ over directed 3-regular graphs $G$, which are \#P-hard in general but become tractable over planar graphs.

The main innovation in this article is the idea of an anti-gadget. Coupled with the idea of antigadgets, we also introduce a general way of proving \#P-hardness by two types of gadgets, called recursive gadgets and projector gadgets. We prove a Group Lemma which spells out a general condition for the technique to succeed. This Group Lemma states that as long as the group generated by the transition matrices of the constructed gadgets is infinite, then one can interpolate all unary functions-a key step in the proof of \#P-hardness. Interpolation is carried out by forming a Vandermonde system and proving that it is of full rank. The anti-gadget concept makes the transition to group theory natural and seamless.

In terms of concrete theorems in complexity theory, this article can be viewed as extending previous dichotomy theorems for the complexity of spin systems with symmetric edge functions [13, $14,19,21,35,36]$ to asymmetric edge functions. The new dichotomy theorem holds over 3-regular graphs, for any (not necessarily symmetric) complex-valued edge function. In physics, the $0-1$ vertex assignments are called spins, and the edge function values $f(\sigma(u), \sigma(v))$ correspond to local interactions between particles. There is a long history in the statistical physics community in the study of "Exactly Solved Models" [3, 38]. In the language of modern complexity theory, physicists'

\footnotetext{
${ }^{1}$ To state our results strictly in Turing's model of computation, we assume $f$ takes algebraic complex values.
} 
notion of an "Exactly Solvable" system roughly corresponds to a system with polynomial-time computable partition function. A central question is to identify which "systems" can be solved "exactly" and which "systems" are "difficult." While in physics there is no rigorous definition of being "difficult," in complexity theory this is formulated as \#P-hardness. The various dichotomies for such partition functions, which divide the whole parameter space into being either polynomialtime computable or \#P-hard, provide evidence that being "difficult" corresponds to being \#P-hard, with nothing being lost in the gap between tractable and difficult.

The class of problems that we study in this article has a close connection with Holant problems $[12,16,18,20,23,32,34,41,42]$. We use holographic algorithms [16, 41] to prove both tractability and \#P-hardness. In general, Holant problems are a natural class of counting problems that can encode all counting Constraint Satisfaction Problems (\#CSP) and graph homomorphisms [24]. Dichotomy theorems for graph homomorphisms [4, 8, 10, 26, 27, 30, 33, 37] and \#CSP [4-6, 9, 12, 22, 25, 28, 29, 31] have been a very active research area. Compared with \#CSP and graph homomorphisms, we still do not have a general dichotomy for Holant problems without a symmetry condition or auxiliary functions $[1,2,7,11,20]$. The main difficulty here is dealing with bounded or fixed-degree graphs. The work reported here is intended as a building block toward that goal.

We remark that some proof in this article relies on Mathematica, in particular, the functions CylindricalDecomposition and Reduce (used in the proof of Lemma 6.10 and Lemma 6.20, respectively). If implemented properly, when CyLINDRICALDECOMPOSITION states that there are no solutions to a particular system, as in the proof of Lemma 6.10, it is a mathematical proof that no solution exists, not merely that it could not find a solution. With the sole exception of CyLINDRICALDECOMPOSITION in the proof of Lemma 6.10, the use of Mathematica in this article can be removed (including the use of REDUCE in the proof of Lemma 6.20). For the convenience of the readers, we have provided some Mathematica resources to verify the validity of parts of some proofs. Mathematica notebooks are available as supplemental material on the website of the journal hosting this article. Transcripts of the executions of those files using Mathematica $11.3 \mathrm{can}$ be found in Appendix B.

\section{NOTATION AND BACKGROUND}

The partition function

$$
Z(G)=\sum_{\sigma: V \rightarrow\{0,1\}} \prod_{(u, v) \in E} f(\sigma(u), \sigma(v))
$$

from Section 1 can be expressed as a Holant problem, a more general framework, defined as follows. A signature grid $\Omega=(G, \mathcal{F}, \pi)$ consists of a labeled undirected graph $G=(V, E)$, where $\pi$ labels each vertex $v \in V$ with a function $f_{v} \in \mathcal{F}$ and the incident edges $E(v)$ at $v$ with the input variables of $f_{v}$. The inputs of $f_{v}$ are identified with $E(v)$. For any edge assignment $\xi$ : $E \rightarrow\{0,1\}, f_{v}\left(\left.\xi\right|_{E(v)}\right)$ is the evaluation at $v$, and the counting problem is to compute Holant s $_{\Omega}=$ $\sum_{\xi: E \rightarrow\{0,1\}} \prod_{v \in V} f_{v}\left(\left.\xi\right|_{E(v)}\right)$.

The partition function $Z(G)$ for any directed 3-regular graph $G=(V, E)$ is a vertex assignment model, where for each directed edge $(u, v)$, an edge function $f$ gives the output value $f(\sigma(u), \sigma(v))$. To express $Z(G)$ as a Holant problem, consider its edge-vertex incidence graph $G^{\prime}$, which has vertex set $V\left(G^{\prime}\right)=V \cup E$ and edge set $E\left(G^{\prime}\right)=\{(v, e) \mid v$ is incident to $e$ in $G\}$. The graph $G^{\prime}$ is bipartite and $(2,3)$-regular. If we label each $v \in V \subset V\left(G^{\prime}\right)$ with the EQUALITy function $=_{3}$ of arity 3 and each $e \in E \subset V\left(G^{\prime}\right)$ with the original edge function $f$ from $G$, then the Holant value on $G^{\prime}$ is exactly the partition function $Z(G)$. Essentially $=_{3}$ forces all incident edges in $G^{\prime}$ at a vertex $v \in V \subset V\left(G^{\prime}\right)$ 


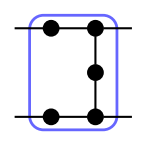

(a) As a bipartite (2,3)-regular graph

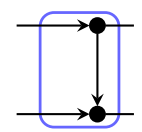

(b) As a directed 3-regular graph

Fig. 1. The two representations of an example $(\mathcal{G} \mid \mathcal{R})$-gate. In (b), a binary constraint $f\left(x_{1}, x_{2}\right)$ is depicted by a directed edge from $x_{1}$ to $x_{2}$.

to take the same value, which reduces to vertex assignments on $V$, as in $Z(G)$. In our proof, we frequently take this bipartite perspective of $Z(G)$ as Holant problems because we use holographic transformations, which are more convenient on bipartite graphs.

A function $f:\{0,1\}^{k} \rightarrow \mathbb{C}$ can be denoted by $\left(f_{0}, f_{1}, \ldots, f_{2^{k}-1}\right)$, where $f_{i}$ is the value of $f$ on the $i$ th lexicographical bit string of length $k$. They are also called signatures. A signature $f$ of arity $k$ is degenerate if $f$ is a tensor product of unary signatures: $f=\left(a_{1}, b_{1}\right) \otimes \cdots \otimes\left(a_{k}, b_{k}\right)$. For $(2,3)$ regular bipartite graphs $(U, V, E)$, if every $u \in U$ is labeled by a binary function $f$ and every $v \in V$ is labeled by a ternary function $g$, then we also use Holant $(f \mid g)$ to denote the Holant problem. Our main result is a dichotomy theorem for Holant $\left(f \mid=_{3}\right)$ for an arbitrary binary function $f=$ $(w, x, y, z)$, where $w, x, y, z \in \mathbb{C}$. It has the same complexity as Holant $\left(c f \mid={ }_{3}\right)$ for any nonzero $c \in \mathbb{C}$; hence, we often normalize a signature by a nonzero scalar. More generally, if $\mathcal{G}$ and $\mathcal{R}$ are finite sets of signatures and the vertices of $U$ (resp., $V$ ) are labeled by signatures from $\mathcal{G}$ (resp., $\mathcal{R}$ ), then we also use Holant $(\mathcal{G} \mid \mathcal{R})$ to denote the bipartite Holant problem. Signatures in $\mathcal{G}$ are called generators and signatures in $\mathcal{R}$ are called recognizers.

Signatures from $\mathcal{G}$ and $\mathcal{R}$ can be used at each vertex of the right degree belonging to the appropriate (left or right) part of a (bipartite) input graph. A $(\mathcal{G} \mid \mathcal{R})$-gate $\Gamma$ is a triple $(H, \mathcal{G}, \mathcal{R})$, where $H=(U, V, E, D)$ is a bipartite graph with some dangling edges $D$. Other than these dangling edges, a $(\mathcal{G} \mid \mathcal{R})$-gate is the same as a signature grid. In $H=(U, V, E, D)$, each node in $U$ (resp., $V$ ) is assigned a function in $\mathcal{G}$ (resp., $\mathcal{R}$ ), $E$ are the ordinary edges, and $D$ are the dangling edges. The purpose of a $(\mathcal{G} \mid \mathcal{R})$-gate is to define a function, and its dangling edges correspond to variables. It defines a function: $\Gamma\left(y_{1}, y_{2}, \ldots, y_{q}\right)=\sum_{\left(x_{1}, x_{2}, \ldots, x_{p}\right) \in\{0,1\}^{p}} H\left(x_{1}, x_{2}, \ldots, x_{p}, y_{1}, y_{2}, \ldots, y_{q}\right)$, where $p=|E|, q=|D|,\left(y_{1}, y_{2}, \ldots, y_{q}\right) \in\{0,1\}^{q}$ denotes an assignment on the dangling edges, and $H\left(x_{1}, x_{2}, \ldots, x_{p}, y_{1}, y_{2}, \ldots, y_{q}\right)$ denotes the product of evaluations at every vertex of $H$. We also call this function the signature of the $(\mathcal{G} \mid \mathcal{R})$-gate $\Gamma$. A $(\mathcal{G} \mid \mathcal{R})$-gate can be used in a signature grid, provided that we observe the bipartite restriction. Signature grids on bipartite $(2,3)$-regular graphs can be identified with directed 3-regular graphs, where we merge two incident edges at every vertex $w$ of degree 2 , and label the new edge by the arity 2 signature $f_{w}$. The edge is oriented from $u$ to $v$ if $f_{w}=f_{w}(u, v)$. Figure 1 gives an example of a $(\mathcal{G} \mid \mathcal{R})$-gate both as a bipartite $(2,3)$-regular graph and as an equivalent directed 3-regular graph.

We designate dangling edges as either leading edges or trailing edges. We require that all leading edges of a $(\mathcal{G} \mid \mathcal{R})$-gate are internally connected to a vertex of the left-hand side with a signature from $\mathcal{G}$, and all trailing edges are internally connected to a vertex of the right-hand side with a signature from $\mathcal{R}$. Each $(\mathcal{G} \mid \mathcal{R})$-gate is pictured with leading edges protruding to the left and any trailing edges to the right. Suppose that a $(\mathcal{G} \mid \mathcal{R})$-gate has $m$ leading edges and $n$ trailing edges. Then the signature of the $(\mathcal{G} \mid \mathcal{R})$-gate can be organized as a $2^{m}$-by- $2^{n}$ transition matrix $M$, where a 


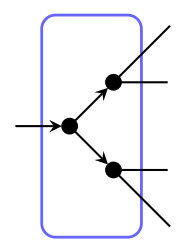

(a)

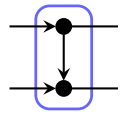

(b)

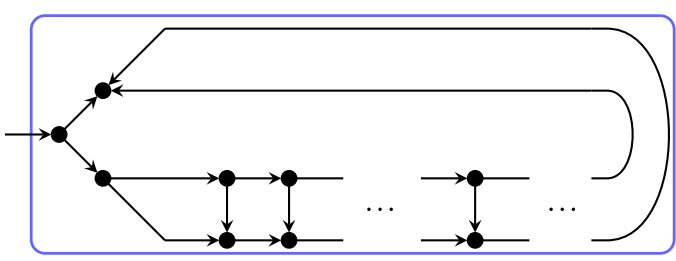

(c)

Fig. 2. An arity 4-to-1 projector gadget (a), a recursive gadget (b), and a planar construction (c) using one copy of (a) and a sequence of (b). Note that following the last copy of (b) there are two extra degree 2 vertices (depicted as two directed edges) connected back to (a), in order to satisfy the bipartite restriction.

row (resp., a column) is indexed by a $\{0,1\}$-assignment to the leading (resp., trailing) edges. When pictured, if $e_{1}, \ldots, e_{n}$ are $n$ dangling edges in top-to-bottom order, then $b_{1} \ldots b_{n} \in\{0,1\}^{n}$ is the index for the assignment where $e_{i}$ is assigned $b_{i}$. We denote the transition matrix of Gadget $i$ as $M_{i}$ unless otherwise noted.

The constructions in this article are primarily based on two kinds of $(\mathcal{G} \mid \mathcal{R})$-gates, which we call recursive gadgets and projector gadgets. An arity-d recursive $(\mathcal{G} \mid \mathcal{R})$-gadget is a $(\mathcal{G} \mid \mathcal{R})$-gate with $d$ leading edges and $d$ trailing edges. A $(\mathcal{G} \mid \mathcal{R})$-gate is a projector $(\mathcal{G} \mid \mathcal{R})$-gadget from arity $n$ to $m$ if it has $m$ leading edges and $n$ trailing edges. For $(\mathcal{G} \mid \mathcal{R})$-gates on a bipartite $(2,3)$-regular graph, internally, for both recursive and projector gadgets, we require that all leading edges connect to a degree 2 vertex (depicted as a directed edge oriented from $x_{1}$ to $x_{2}$ if the binary function is $f\left(x_{1}, x_{2}\right)$ ), while all trailing edges connect to a degree 3 vertex (depicted as an undirected edge, which is externally to be merged with a directed edge, i.e., a degree 2 vertex). These gadget types are defined in this way to maintain the bipartite structure of the signature grid when we merge trailing edges of one gadget with leading edges of another (Figure 2).

\section{GADGETS AND ANTI-GADGETS}

In this section, we start with a gentle primer on the association between a combinatorial gadget and its signature written as a transition matrix. We show that one can typically express the transition matrix starting from a few of the most basic gadget components and their matrices as atomic building blocks after applying some well-defined operations. We then introduce anti-gadgets and explain why they are so effective.

We start with five basic gadget components, as depicted in Figure 3. Their signature matrices are

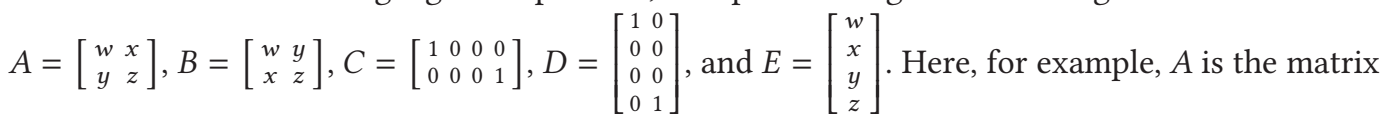
of a binary signature on $\left(x_{1}, x_{2}\right) \in\{0,1\}^{2}$, and $x_{1}$ (resp., $\left.x_{2}\right)$ is the row (resp., column) index.

The first operation is matrix product, which corresponds to sequentially connecting two gadgets. For example, Gadget 1 is a simple composition of Gadget B and Gadget C; thus, its transition matrix is the matrix product $B C=\left[\begin{array}{llll}w & 0 & 0 & y \\ x & 0 & 0 & z\end{array}\right]$ (see Figure $5(\mathrm{a})$ ). The second operation is 


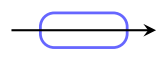

Gadget A

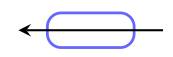

Gadget B

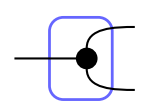

Gadget C

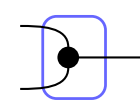

Gadget D
J

Gadget E

Fig. 3. Five basic gadget components.

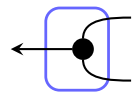

Gadget 1

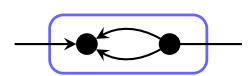

Gadget 2

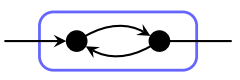

Gadget 3

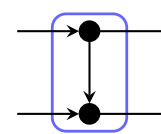

Gadget 4

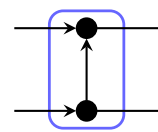

Gadget 5

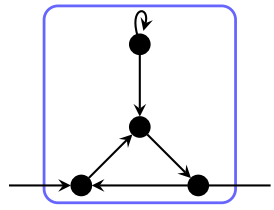

Gadget 6

Fig. 4. Recursive and projector gadgets.

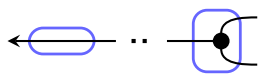

(a) Composition of Gadget 1

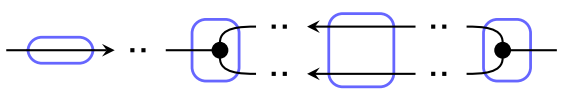

(b) Composition of Gadget 2

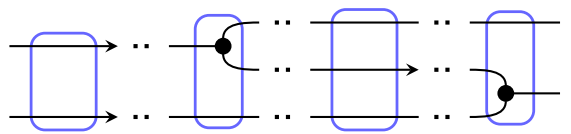

(c) Composition of Gadget 4

Fig. 5. Gadget compositions using the basic gadget components in Figure 3.

tensor product, which corresponds to putting two gadgets in parallel (two disconnected parts). The transition matrix of Gadget 2 is $A C B^{\otimes 2} D=\left[\begin{array}{cc}w^{3}+x^{3} & w y^{2}+x z^{2} \\ w^{2} y+x^{2} z & y^{3}+z^{3}\end{array}\right]$, where $B^{\otimes 2}$ corresponds to the parallel part of the gadget and is clearly visible in Figure 5(b). Similarly, Gadget 3 has signature matrix $A C(A \otimes B) D$. One can also write the transition matrices of Gadget 2 and Gadget 3 as $\left[\begin{array}{ll}w & x \\ y & z\end{array}\right]\left[\begin{array}{ll}w^{2} & y^{2} \\ x^{2} & z^{2}\end{array}\right]$ and $\left[\begin{array}{ll}w & x \\ y & z\end{array}\right]\left[\begin{array}{ll}w^{2} & x y \\ x y & z^{2}\end{array}\right]$, respectively. Note that the order of the tensor product is to make the top leading edge for the row (resp., the top trailing edge for the column) the most significant bit. The transition matrices of Gadget 4 and Gadget 5 are, respectively, $\left[\begin{array}{ll}w & x \\ y & z\end{array}\right]^{\otimes 2} \operatorname{diag}(w, x, y, z)$ and $\left[\begin{array}{ll}w & x \\ y & z\end{array}\right]^{\otimes 2} \operatorname{diag}(w, y, x, z)$ and can be mechanically derived by our gadgetry calculus as $A^{\otimes 2}\left(C \otimes I_{2}\right)\left(I_{2} \otimes A \otimes I_{2}\right)\left(I_{2} \otimes D\right)$ and $A^{\otimes 2}\left(C \otimes I_{2}\right)\left(I_{2} \otimes B \otimes I_{2}\right)\left(I_{2} \otimes D\right)$. The composition of Gadget 4 is illustrated in Figure 5(c). Gadget $\mathrm{E}$ is used to create a self-loop, as in Gadget 6, which has transition matrix $A C\left(A \otimes I_{2}\right)\left(C \otimes I_{2}\right)\left(B C E \otimes I_{4}\right)(A \otimes B) D$. A composition is given in Figure 6.

Now, we introduce a new technique called anti-gadgets. 


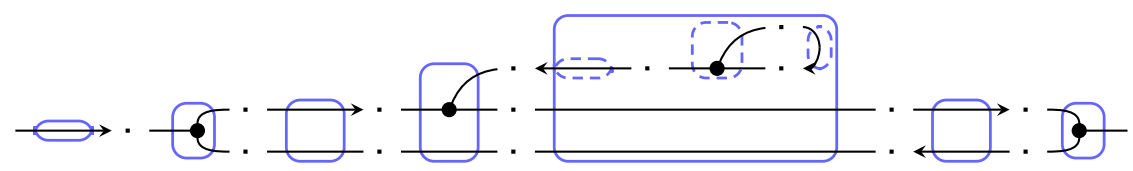

Fig. 6. Composition of Gadget 6 using the basic gadget components in Figure 3.

Definition 3.1. Let $G$ be a recursive gadget with transition matrix $M$. Then, a recursive gadget $G^{\prime}$ is called an anti-gadget of $G$ if the transition matrix of $G^{\prime}$ is $\lambda M^{-1}$ for some $\lambda \in \mathbb{C}-\{0\}$.

A crucial ingredient in our proof of \#P-hardness is to produce an infinite set of pairwise linearly independent signatures. For each problem instance, a finite number of these signatures is used to form a Vandermonde system of full rank. The exact number of signatures required grows at most polynomially with the size of the instance. One common way to produce such an infinite set of signatures is to compose copies of a recursive gadget. Let $M$ be the transition matrix of some recursive gadget $G$. Suppose that $M$ is an invertible matrix. As discussed above, composing $k$ copies of $G$ produces a gadget with transition matrix $M^{k}$. If $M$ has infinite order (up to a scalar), that is, for all integers $k>0, M^{k}$ is not a scalar matrix (a linear multiple of $I$ ), then we have an infinite set of pairwise linearly independent signatures, and any instance for the reduction proof can use polynomially many of these signatures. Indeed, if $M^{k}$ and $M^{\ell}$ are linearly dependent for some $k \neq \ell$, then for $k^{\prime}=|k-\ell|, M^{k^{\prime}}$ is $\lambda I$ for some $\lambda$. Now, suppose that $M$ has finite order (up to a scalar), that is, for some integer $k>0, M^{k}=\lambda I$. Then, composing only $k-1$ copies of $G$ results in a gadget with a transition matrix that is the inverse of $G$ 's transition matrix (up to a scalar). For $k=1$, the statement is also valid. This is an anti-gadget of $G$.

If an anti-gadget of $G$ is composed with another gadget containing a similar structure to that of $G$, then cancellations ensue and the composition yields a transition matrix that can be quite easy to analyze. For example, Gadget 4 and Gadget 5 differ only by the orientation of the vertical edge. When composing an anti-gadget of Gadget 4 with Gadget 5, the contribution of the two leading edges cancel and we get that $M_{4}^{-1} M_{5}=\operatorname{diag}(w, x, y, z)^{-1}\left(\left[\begin{array}{ll}w & x \\ y & z\end{array}\right]^{\otimes 2}\right)^{-1}\left[\begin{array}{ll}w & x \\ y & z\end{array}\right]^{\otimes 2} \operatorname{diag}(w, y, x, z)=$ $\operatorname{diag}(1, y / x, x / y, 1)$. The resulting transition matrix has infinite order unless $x / y$ is a root of unity. This situation is analyzed formally in Lemma 6.1.

Another use of the anti-gadget technique can be applied with Gadget 2 and Gadget 3. Once again, the contribution of the leading edge cancels when composing an anti-gadget of Gadget 3 with Gadget 2. The resulting matrix is a bit more complicated this time. However, when this pair of gadgets is analyzed formally in Lemma 6.2, the assumptions are that $x=0 \wedge w y z \neq 0$. In that case, $M_{3}^{-1} M_{2}=\left[\begin{array}{cc}1 & y^{2} / w^{2} \\ 0 & 1\end{array}\right]$. This matrix clearly has infinite order (up to a scalar).

\section{PROJECTIVE SETS AND THE GROUP LEMMA}

The method of polynomial interpolation has been pioneered by Valiant [40] and further developed by many others [4, 6, 21, 27, 39]. In this section, we present a new unified technique to interpolate all unary signatures. This is our main technical step to prove \#-hardness. Our method produces an infinite set of pairwise linearly independent vectors at any fixed dimension and then projects to a lower dimension while retaining pairwise linear independence of a nontrivial fraction.

In previous work, "finisher gadgets" [13, 14, 35, 36] were used to map arity 2 signatures to arity 1 signatures. However, they handle only the symmetric case. In the present work, we introduce projective gadget sets. These gadget sets can be used to map any set of pairwise linearly independent signatures (symmetric or asymmetric) from any higher arity to a lower arity, while preserving pairwise linear independence for an inverse polynomial fraction. This permits much 
more freedom in gadget constructions compared with the "finisher gadgets," and this power is used crucially in the proof of our dichotomy theorem. We remark that this advance is not just a simple matter of searching for the right gadgets. One must find the abstract criteria for success that can be simultaneously satisfied by gadgets that actually exist. These developments, together with the anti-gadget concept, come together in the Group Lemma, which provides a straightforward criterion for proving \#P-hardness of certain Holant problems.

Definition 4.1. A set of matrices $\mathcal{M} \subseteq \mathbb{C}^{2^{m} \times 2^{n}}$ forms a projective set from arity $n$ to $m$ if for any matrix $N \in \mathbb{C}^{2^{n} \times 2}$ with rank 2 , there exists a matrix $M \in \mathcal{M}$ such that $M N$ has rank 2 .

We also call a set of gadgets projective from arity $n$ to $m$ if the set of its signature matrices is projective from arity $n$ to $m$. A gadget set that is projective from arity 2 to 1 can be used to transform a pair of $(\mathcal{G} \mid \mathcal{R})$-gates with linearly independent binary signatures to a pair of $(\mathcal{G} \mid \mathcal{R})$-gates with linearly independent unary signatures (provided that the bipartite condition is satisfied). Projector gadgets from arity 2 to 1 in such a set have two trailing edges and one leading edge. But they can also be viewed as operating on signatures of higher arity, with the identity transformation (expressed as a tensor product with $I$ ) being performed on the other edges not connected to the projector gadget (depicted as parallel edges). This way of connecting the projector gadget to an existing $(\mathcal{G} \mid \mathcal{R})$-gate automatically gives us projective gadget sets for higher arities. But first, a quick lemma to assist with the proof.

LEMMA 4.2. Let $u$ and $v$ be nonzero column vectors, not necessarily of the same length. Then, the block matrix $\left[\begin{array}{ll}a u & b u \\ c v & d v\end{array}\right]$ has rank 2 if and only if $\left[\begin{array}{ll}a & b \\ c & d\end{array}\right]$ is invertible.

Proof. We write $\left[\begin{array}{ll}a u & b u \\ c v & d v\end{array}\right]=\left[\begin{array}{ll}u & 0 \\ 0 & v\end{array}\right]\left[\begin{array}{ll}a & b \\ c & d\end{array}\right]$, and $\left[\begin{array}{ll}u & 0 \\ 0 & v\end{array}\right]$ has rank 2. Thus, $\left[\begin{array}{ll}a u & b u \\ c v & d v\end{array}\right]$ has rank 2 if and only if $\left[\begin{array}{ll}a & b \\ c & d\end{array}\right]$ is invertible.

Lemma 4.3. Let $\mathcal{G}$ and $\mathcal{R}$ be signature sets. Suppose that $\mathcal{P}$ is a projective $(\mathcal{G} \mid \mathcal{R})$-gate set from arity 2 to 1 . Then, for all integers $k \geq 2, \mathcal{P}$ acts as a projective $(\mathcal{G} \mid \mathcal{R})$-gate set from arity $k$ to $k-1$.

Proof. We are given that for any $N \in \mathbb{C}^{4 \times 2}$ with rank 2, there exists an $F \in \mathcal{P}$ with $F \in \mathbb{C}^{2 \times 4}$ such that $F N$ is invertible. We want to show that for any integer $k \geq 2$ and any rank 2 matrix $B \in \mathbb{C}^{2^{k} \times 2}$, there exists an $F \in \mathcal{P}$ such that $(I \otimes F) B$ has rank 2 , where $I$ is the $2^{k-2}$-by-2 $2^{k-2}$ identity matrix.

For any $F \in \mathcal{P}$, the matrix $I \otimes F$ can be viewed as being composed of 2-by-4 blocks, with $F$ appearing along the main diagonal and 2-by-4 zero-matrices elsewhere. We similarly view $B$ as being composed of 4-by-2 blocks $B=\left[\begin{array}{c}B_{1} \\ B_{2} \\ \vdots \\ B_{2} k-2\end{array}\right]$. Then, $(I \otimes F) B=\left[\begin{array}{c}F B_{1} \\ F B_{2} \\ \vdots \\ F B_{2 k-2}\end{array}\right] \in \mathbb{C}^{2^{k-1} \times 2}$. If some $B_{i}$ has rank 2, then there is an $F \in \mathcal{P}$ such that $F B_{i}$ is invertible and $(I \otimes F) B$ has rank 2, as desired.

Now, assume otherwise, so that each $B_{i}$ has rank at most 1 . Since $B$ has rank 2, there exists a 2-by-2 invertible submatrix $D$ of $B$, for which the rows of $D$ appear in $B_{i}$ and $B_{j}$ for some $i<j$. It follows that $B_{i}$ and $B_{j}$ both have rank exactly 1 . Hence, for some nonzero vectors $u, v \in \mathbb{C}^{4}$

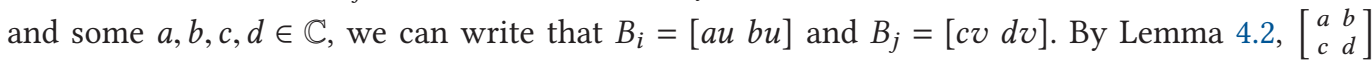
is invertible, as $\left[\begin{array}{l}B_{i} \\ B_{j}\end{array}\right]$ has rank 2 . If $u$ and $v$ are linearly independent, then choose $F \in \mathcal{P}$ such that $F[u v]$ is invertible. Otherwise, let $\tilde{v} \in \mathbb{C}^{4}$ be such that $u$ and $\tilde{v}$ are linearly independent, and choose $F \in \mathcal{P}$ such that $F[u \tilde{v}]$ is invertible. In either case (ignoring $\tilde{v}$ in the second case), we define $\left[\begin{array}{ll}u^{\prime} & v^{\prime}\end{array}\right]=F\left[\begin{array}{ll}u & v\end{array}\right]$, where $u^{\prime}$ and $v^{\prime}$ are nonzero. Then, by Lemma 4.2, the matrix $\left[\begin{array}{ll}a u^{\prime} & b u^{\prime} \\ c v^{\prime} & d v^{\prime}\end{array}\right]=\left[\begin{array}{l}F B_{i} \\ F B_{j}\end{array}\right]$ has rank 2, and since this appears as a submatrix of $(I \otimes F) B$, we are done. 
Corollary 4.4. Let $\mathcal{G}$ and $\mathcal{R}$ be signature sets. Suppose that $\mathcal{P}$ is a finite projective $(\mathcal{G} \mid \mathcal{R})$-gate set from arity 2-to-1. Then, for any integer $k \geq 2, \mathcal{P}$ induces a finite projective $(\mathcal{G} \mid \mathcal{R})$-gate set from arity $k$ to 1.

Now, we show that a finite projective $(\mathcal{G} \mid \mathcal{R})$-gadget set from arity $k$ to 1 preserves pairwise linear independence for an inverse polynomial fraction of signatures. The essence of the next lemma is an exchange in the order of quantifiers.

Lemma 4.5. Suppose that $\left\{v_{i}\right\}_{i \geq 0}$ is an infinite sequence of pairwise linearly independent column vectors in $\mathbb{C}^{2^{k}}$ and let $\mathcal{F} \subseteq \mathbb{C}^{2 \times 2^{k}}$ be a finite set of $t$ matrices that is projective from arity $k$ to 1 . Then, for every $n$, there exists some $F \in \mathcal{F}$ and some $S \subseteq\left\{F v_{i} \mid 0 \leq i \leq n^{t}\right\}$ such that $|S| \geq n$ and the vectors in $S$ are pairwise linearly independent.

Proof. Let $j>i \geq 0$ be integers and let $N=\left[v_{i} v_{j}\right] \in \mathbb{C}^{2^{k} \times 2}$. Since $v_{i}$ and $v_{j}$ are linearly independent, $\operatorname{rank}(N)=2$. By assumption, there exists an $F \in \mathcal{F}$ such that $F N \in \mathbb{C}^{2 \times 2}$ is invertible; thus, we conclude that $F v_{i}$ and $F v_{j}$ are linearly independent.

Each $F \in \mathcal{F}$ defines a coloring of the set $K=\left\{0,1, \ldots, n^{t}\right\}$ as follows: color $i \in K$ with the linear subspace spanned by $F v_{i}$. Assume for a contradiction that for each $F \in \mathcal{F}$, there are no $n$ pairwise linearly independent vectors among $\left\{F v_{i} \mid i \in K\right\}$. Then, including possibly the 0 -dimensional subspace $\{0\}$, there can be at most $n$ distinct colors assigned to $K$ by each $F \in \mathcal{F}$. Since $|\mathcal{F}|=t$, by the pigeonhole principle, some $i$ and $j$ with $0 \leq i<j \leq n^{t}$ must receive the same color for all $F \in \mathcal{F}$. This is a contradiction with the previous paragraph; thus, we are done.

The next lemma says that under suitable conditions, we can construct all unary signatures $(X, Y)$. The method will be to collect a sufficiently large polynomial set of pairwise linearly independent signatures at a higher dimension and then use an appropriate projector gadget.

Lemma 4.6 (Group Lemma). For signature sets $\mathcal{G}$ and $\mathcal{R}$, suppose that there exists a finite set of projective $(\mathcal{G} \mid \mathcal{R})$-gates from arity 2 to 1 , and suppose that $\mathcal{S}$ is a finite set of recursive $(\mathcal{G} \mid \mathcal{R})$-gates of arity $d \geq 1$ with nonsingular transition matrices. Let $H$ be the group generated by the transition matrices of gadgets in $\mathcal{S}$, modulo scalar matrices $\lambda I$, for $\lambda \in \mathbb{C}-\{0\}$. If $H$ has infinite order, then

$$
\operatorname{Holant}(\mathcal{G} \cup\{(X, Y)\} \mid \mathcal{R}) \leq_{T} \operatorname{Holant}(\mathcal{G} \mid \mathcal{R})
$$

for any $X, Y \in \mathbb{C}$.

Proof. We first prove a weaker version by making two additional assumptions: (1) $H$ contains some element of infinite order; and (2) $\mathcal{G}$ contains a nondegenerate binary signature $g$. This simplifies the presentation of the proof. After that. we indicate the modifications to the proof, removing the two additional assumptions.

Two matrices from $\mathrm{GL}\left(2^{d}, \mathbb{C}\right)$ are unequal as elements in the projective general linear group $\operatorname{PGL}\left(2^{d}, \mathbb{C}\right)=\mathrm{GL}\left(2^{d}, \mathbb{C}\right) /\{\lambda I \mid \lambda \in \mathbb{C}-\{0\}\}$ if and only if they are linearly independent over $\mathbb{C}$. If the signature matrix of any member of $\mathcal{S}$, as a group element in $H$, has infinite order, then its powers supply an infinite set of pairwise linearly independent signatures. Otherwise, they all have finite order, and the group $H$ is identical to the monoid generated by signature matrices from $\mathcal{S}$, that is, every $h \in H$ is a product of signature matrices from $\mathcal{S}$ with non-negative powers. Each such product defines a recursive gadget of arity $d$ as a sequential composition of gadgets in $\mathcal{S}$. By our additional assumption (1), $H$ contains some element $h$ of infinite order. Then, the powers of $h$ supply an infinite set $\left\{h^{i} \mid i \geq 0\right\}$ of pairwise linearly independent signatures.

Before we can use a projective gadget set to project these signatures $\left\{h^{i}\right\}_{i \geq 0}$, we make a small modification to the gadget of $h^{i}$ for each $i$ : By our additional assumption (2), there is a nondegenerate binary signature $g \in \mathcal{G}$. We connect a degree 2 vertex labeled with $g$ to every trailing 
edge of $h^{i}$. This ensures that the bipartite structure of the graph is preserved when applying projector gadgets. Let $M$ be the 2-by-2 matrix of $g$. As there are $d$ trailing edges, we apply $d$ copies of $g$, which corresponds to multiplication by the matrix $M^{\otimes d}$. Since $M$ has full rank, pairwise linear independence of the signatures is preserved. We rewrite the $2^{d}$-by- $2^{d}$ matrix form of the signature $h^{i} M^{\otimes d}$ as a column vector $v_{i} \in \mathbb{C}^{2 d}$, indexed by $c_{d} \cdots c_{1} b_{1} \cdots b_{d} \in\{0,1\}^{2 d}$, where $b_{1} \cdots b_{d}$ and $c_{1} \cdots c_{d}$ are the row and column indices. Now, we can attach a projector gadget to project each $v_{i}$ down to arity 1 (see Figure 2(c), which illustrates the case for $d=2$ ).

By Corollary 4.4, there exists a finite projective set $\mathcal{P}$ of gadgets from arity $2 d$ to 1 . Let $t=|\mathcal{P}|$. For every $n \geq 1$, we can produce in polynomial time $(n+2)^{t}+1$ vectors $\left\{v_{i} \mid 0 \leq i \leq(n+2)^{t}\right\}$ as described above, and there exists some $F \in \mathcal{P}$ such that the set $\left\{F v_{i} \mid 0 \leq i \leq(n+2)^{t}\right\}$ contains some subset $S$ of pairwise linearly independent vectors, where $|S| \geq n+2$ by Lemma 4.5 . Such a subset can be found in polynomial time greedily. For every $F \in \mathcal{P}$, we pick the first nonzero vector of the form $F v_{i}$ (if there is a nonzero vector of this form) and sequentially pick the rest of the subset as follows. After having picked the first $k \geq 1$ vectors of the form $F v_{i}$ that are pairwise linearly independent, if $k<n+2$, then we include the next $F v_{i}$ if and only if it is not a linear multiple of any of the $k$ vectors picked so far. Suppose that $F$ and $S$ satisfy Lemma 4.5. We claim that the above process will produce some subset $S^{\prime}$ of cardinality $\left|S^{\prime}\right| \geq n+2$. Otherwise, suppose that we end the process with some $S^{\prime}$ of cardinality $\left|S^{\prime}\right|<n+2$. Since some nonzero vector of the form $F v_{i}$ exists, $S^{\prime}$ is nonempty. Every vector of $S$ is a linear multiple of some vector in $S^{\prime}$, because it is either placed in $S^{\prime}$ or found to be a linear multiple (no vector in $S$ is the zero vector by pairwise linear independence) of a vector already in $S^{\prime}$. As $\left|S^{\prime}\right|<|S|$, two distinct vectors of $S$ are both linear multiples of some vector in $S^{\prime}$. This is a contradiction to pairwise linear independence of $S$. We also note that we do not need to know which $F$ works; if for more than one $F$ the above process produced a subset of cardinality $\geq n+2$, there is no harm in what follows.

To show Holant $(\mathcal{G} \cup\{(X, Y)\} \mid \mathcal{R}) \leq_{T} \operatorname{Holant}(\mathcal{G} \mid \mathcal{R})$, suppose that we are given as input a bipartite signature grid $\Omega$ for Holant $(\mathcal{G} \cup\{(X, Y)\} \mid \mathcal{R})$, with underlying graph $G=(V, E)$. Let $Q \subseteq V$ be the set of vertices labeled with generator $(X, Y)$, and let $n=|Q|$. We can obtain a set of pairwise linearly independent unary signatures $\left\{\left(X_{0}, Y_{0}\right),\left(X_{1}, Y_{1}\right), \ldots,\left(X_{n+1}, Y_{n+1}\right)\right\}$ in polynomial time as described above. Let $G_{0}, G_{1}, \ldots, G_{n+1}$ be the corresponding gadgets. There is a polynomial $p(\cdot)$ such that all $G_{0}, G_{1}, \ldots, G_{n+1}$ have size at most $p(n)$. (This polynomial is uniform for all signature grids $\Omega$.) At most one $Y_{\ell}$ can be zero; thus, without loss of generality, assume that $Y_{\ell} \neq 0$ for $0 \leq \ell \leq n$. If we replace every element of $Q$ with a copy of $G_{\ell}$, we obtain an instance of $\operatorname{Holant}(\mathcal{G} \mid \mathcal{R}$ ) (note that the correct bipartite structure is preserved). We denote this new signature grid by $\Omega_{\ell}$.

Although Holant $\Omega$ is a sum of exponentially many terms, all terms can be classified according to the exact number of times $j(0 \leq j \leq n)$ where the signature $(X, Y)$ on a vertex in $Q$ is assigned 0 . Then, for some $c_{0}, c_{1}, \ldots, c_{n} \in \mathbb{C}$ (not depending on the values $X$ and $Y$ ), we have that

$$
\text { Holant }_{\Omega}=\sum_{0 \leq j \leq n} c_{j} X^{j} Y^{n-j}
$$

Similarly, for the same values $c_{0}, c_{1}, \ldots, c_{n} \in \mathbb{C}$, we have that

$$
\text { Holant }_{\Omega_{\ell}}=\sum_{0 \leq j \leq n} c_{j} X_{\ell}^{j} Y_{\ell}^{n-j} .
$$

Since each signature grid $\Omega_{\ell}$ is an instance of $\operatorname{Holant}(\mathcal{G} \mid \mathcal{R})$ of polynomial size, Holant $\Omega_{\ell}$ can be solved exactly using the oracle. We arrive at a linear system in which the $c_{j}$ values are 
the unknowns.

$$
\left[\begin{array}{c}
Y_{0}^{-n} \cdot \text { Holant }_{\Omega_{0}} \\
Y_{1}^{-n} \cdot \text { Holant }_{\Omega_{1}} \\
\vdots \\
Y_{n}^{-n} \cdot \text { Holant }_{\Omega_{n}}
\end{array}\right]=\left[\begin{array}{cccc}
X_{0}^{0} Y_{0}^{0} & X_{0}^{1} Y_{0}^{-1} & \cdots & X_{0}^{n} Y_{0}^{-n} \\
X_{1}^{0} Y_{1}^{0} & X_{1}^{1} Y_{1}^{-1} & \cdots & X_{1}^{n} Y_{1}^{-n} \\
\vdots & \vdots & \ddots & \vdots \\
X_{n}^{0} Y_{n}^{0} & X_{n}^{1} Y_{n}^{-1} & \cdots & X_{n}^{n} Y_{n}^{-n}
\end{array}\right]\left[\begin{array}{c}
c_{0} \\
c_{1} \\
\vdots \\
c_{n}
\end{array}\right]
$$

The matrix above has entry $\left(X_{\ell} / Y_{\ell}\right)^{c}$ at row $\ell$ and column $c$. Due to pairwise linear independence of $\left(X_{\ell}, Y_{\ell}\right)$, the values in the set $\left\{X_{\ell} / Y_{\ell} \mid 0 \leq \ell \leq n\right\}$ are pairwise distinct. Hence, this is a Vandermonde system of full rank, and we can solve it for the $c_{j}$ values. With these values in hand, we can calculate Holant ${ }_{\Omega}$ directly, completing the reduction.

Now, we remove the two additional assumptions. We may still assume that every element of $H$ has a finite order. Thus, group $H$ is the same as the monoid generated by the finite set $\mathcal{S}$. Every element of $H$ can be expressed as a product of matrices from $\mathcal{S}$. Since $H$ is infinite, the Cayley graph of $H$ is infinite. Every node is reachable by a path labeled by a product from $\mathcal{S}$. Such products define recursive gadgets as compositions of gadgets in $\mathcal{S}$. Since distinct nodes of the Cayley graph correspond to distinct group elements in $H$, they supply an infinite set of recursive gadgets having pairwise linearly independent signatures. One can obtain polynomially many such signatures in polynomial time, using breadth-first search. (From a finite subset of known nodes of the Cayley graph, using matrix multiplication we check whether following an edge labeled by some $s \in \mathcal{S}$ from an existing node leads to a newly discovered node.) Thus, these recursive gadgets can play the role of $\left\{h^{i} \mid i \geq 0\right\}$ in the proof above.

Now, we deal with the second additional assumption. We claim that there exists some nondegenerate signature $g \in \mathcal{G}$, but being binary is not necessary. If every $g \in \mathcal{G}$ is degenerate, then any recursive gadget $s \in \mathcal{S}$ (note that $\mathcal{S}$ is nonempty; otherwise, $H$ is not infinite) could be rewritten with all leading edges internally incident to unary signatures. The matrix of such a gadget is expressible as a product of a column vector and a row vector (by partitioning $s$ into two gadgets with no shared edges). Hence, the transition matrix of $s$ would have rank at most 1 , which is less than $2^{d}$ as promised. Let $a \geq 2$ be the arity of $g$. One can show by induction that any nondegenerate signature has at least one index $i$, such that if we express the signature as a 2-by- $2^{a-1}$ matrix $M$ indexed by the $i$ th variable for the row and the remaining $a-1$ variables for the column, then $M$ has rank 2 . Choose such a variable of $g$. As there are $d$ trailing edges in $s$, we apply $d$ copies of $g$, connecting the chosen variable of $g$ to each trailing edge of $s$. This corresponds to multiplication (on the right) by the matrix $M^{\otimes d}$. Since $M$ has full rank, $M^{\otimes d}$ has a $2^{d}$-by-2 $2^{d}$ submatrix of rank $2^{d}$ and, thus, the pairwise linear independence of the signatures is preserved. Now, rewrite the $2^{d}-$ by- $2^{d(a-1)}$ matrix form of the signature as a column vector in $\mathbb{C}^{2^{d a}}$, indexed by $c_{d(a-1)} \cdots c_{1} b_{1} \cdots b_{d} \in\{0,1\}^{d a}$, where $b_{1} \cdots b_{d}$ and $c_{1} \cdots c_{d(a-1)}$ are the row and column indices. Denote these vectors as $\left\{v_{i}\right\}_{i \geq 0}$. Finally, we can attach projector gadgets to project each $v_{i}$ down to arity 1 . The rest of the proof is the same.

Here is how we realize a projective set of gadgets from arity 2 to 1 .

LEMMA 4.7. Let $\Phi_{i} \in \mathbb{C}^{2 \times 4}$ for $1 \leq i \leq 7$ be matrices with the following properties: For some $u, v, u_{i}, v_{i}, s, t \in \mathbb{C}^{4}$,

- $\operatorname{ker}\left(\Phi_{i}\right)=\operatorname{span}\left\{u, u_{i}\right\}$ for $1 \leq i \leq 3$,

- $\operatorname{ker}\left(\Phi_{i+3}\right)=\operatorname{span}\left\{v, v_{i}\right\}$ for $1 \leq i \leq 3$,

- $\operatorname{ker}\left(\Phi_{7}\right)=\operatorname{span}\{s, t\}$, and

- $\operatorname{dim} \operatorname{span}\left\{u, u_{1}, u_{2}, u_{3}\right\}=\operatorname{dim} \operatorname{span}\left\{v, v_{1}, v_{2}, v_{3}\right\}=\operatorname{dim} \operatorname{span}\{u, v, s, t\}=4$.

Then, $\left\{\Phi_{i} \mid 1 \leq i \leq 7\right\}$ is a projective set from arity 2 to 1 . 
Table 1. Projectors Used in Each Case (and the Role of Each

Projector within Each Case) in the Proof of Lemma 4.8

\begin{tabular}{|c|c|c|c|c|c|c|c|}
\hline & $\Phi_{1}$ & $\Phi_{2}$ & $\Phi_{3}$ & $\Phi_{4}$ & $\Phi_{5}$ & $\Phi_{6}$ & $\Phi_{7}$ \\
\hline Case 1 & \multirow{5}{*}{$F_{28}$} & $F_{35}$ & \multirow{2}{*}{$F_{37}$} & \multirow{5}{*}{$F_{28}$} & \multirow{5}{*}{$F_{29}$} & $F_{31}$ & \multirow{2}{*}{$F_{33}$} \\
\hline Case 2 & & \multirow{3}{*}{$F_{30}$} & & & & $F_{36}$ & \\
\hline Case 3 & & & $F_{42}$ & & & $F_{41}$ & $F_{34}$ \\
\hline Case 4 & & & & & & $F_{38}$ & \\
\hline Case 5 & & $F_{39}$ & $F_{40}$ & & & $F_{31}$ & $F_{32}$ \\
\hline
\end{tabular}

The seven $\Phi_{i}$ refer to the matrices in Lemma 4.7. As an example, the projective set in Case 1 is the ordered multiset $\left\{F_{28}, F_{35}, F_{37}, F_{28}, F_{29}, F_{31}, F_{33}\right\}$. Note that $F_{28}$ plays the role of both $\Phi_{1}$ and $\Phi_{4}$

Proof. Let $N \in \mathbb{C}^{4 \times 2}$ be a rank 2 matrix with image $(N)=\operatorname{span}\left\{w_{1}, w_{2}\right\}$. If we have that $\operatorname{image}(N)=\operatorname{span}\{u, v\}$, then $\Phi_{7} N$ has rank 2. Otherwise, either $\left\{w_{1}, w_{2}, u\right\}$ or $\left\{w_{1}, w_{2}, v\right\}$ is linearly independent. Say that $\left\{w_{1}, w_{2}, u\right\}$ is linearly independent. The other case is similar. Then, $\left\{w_{1}, w_{2}, u\right\}$ can be further augmented by some $u_{i}$ for $1 \leq i \leq 3$ to form a basis, in which case $\Phi_{i} N$ has rank 2 .

Verifying that a specific set of gadgets forms a projective set from arity 2 to 1 requires only a straightforward linear algebra computation. The next lemma proves that finite projective $\left(f \mid={ }_{3}\right)$ gate sets from arity 2 to 1 exist in most cases. Note that the exceptional cases are either symmetric signatures (for which a dichotomy has been proved $[35,36]$ ) or largely correspond to tractable cases.

Lemma 4.8. Let $w, x, y, z \in \mathbb{C}$ and $f=(w, x, y, z)$. Then, there exists a finite projective $\left(f \mid={ }_{3}\right)$-gate set from arity 2 to 1 unless

$$
[x=y] \vee[w z=x y] \vee[(w, z)=(0,0)] \vee[(x, y)=(0,0)] \vee\left[\left(w^{3}=-z^{3}\right) \wedge(x=-y)\right] .
$$

Proof. Suppose that the exceptional cases do not apply:

$$
[x \neq y] \wedge[w z \neq x y] \wedge[(w, z) \neq(0,0)] \wedge[(x, y) \neq(0,0)] \wedge\left[\left(w^{3} \neq-z^{3}\right) \vee(x \neq-y)\right] .
$$

We prove Lemma 4.8 by exhibiting projective gadget sets that satisfy the hypotheses of Lemma 4.7. Let $F_{i}$ be the transition matrix of Gadget $i$ for $28 \leq i \leq 42$, shown in the Appendix (Figure 14). By the symmetry of switching 0 with 1 , which switches $w$ and $z$, and switches $x$ with $y$, we divide all $(w, x, y, z)$ into five cases:

(1) $w z \neq x y \wedge w x y z \neq 0 \wedge w^{3} x+w x y z+w^{2} z^{2}+y z^{3} \neq 0 \wedge x^{2} \neq y^{2}$,

(2) $w z \neq x y \wedge w x y z \neq 0 \wedge w^{3} x+w x y z+w^{2} z^{2}+y z^{3} \neq 0 \wedge x=-y \wedge w^{3} \neq-z^{3}$,

(3) $w z \neq x y \wedge w x y z \neq 0 \wedge w^{3} x+w x y z+w^{2} z^{2}+y z^{3}=0 \wedge x \neq y$,

(4) $w z \neq x y \wedge w=0 \wedge z \neq 0 \wedge x \neq y$, and

(5) $w z \neq x y \wedge x=0 \wedge y \neq 0$.

This division will be justified shortly. For example, after Case 3, we have addressed the subset $w x y z \neq 0$ within the hypothesized region; by the symmetry of switching 0 and 1 , Cases 4 and 5 complete the picture. In each of the five cases, we exhibit an explicit projective $\left(f \mid==_{3}\right)$-gadget set from arity 2-to-1. We omit the verification that each set of projectors forms a projective gadget set from arity 2-to-1 under its particular assumptions since this is a straightforward linear algebra computation.

Table 1 summarizes which projectors are used in each case and the role of each projector within each case. 
In all five cases, the vector $u$ in the kernels of $\Phi_{1}, \Phi_{2}$, and $\Phi_{3}$ is $(0,-1,1,0)^{\top}$ and the vector $v$ in the kernels of $\Phi_{4}, \Phi_{5}$, and $\Phi_{6}$ is $(0,-x, y, 0)^{\top}$.

All five cases use the assumption that $w z \neq x y$, that is, the edge signature is non-degenerate. Under three additional disequality assumptions stated in Case 1, the projectors in row 1 of Table 1 have the desired properties. The purpose of the remaining four cases is to handle the situation that these three disequalities are not all true.

Case 2 retains two of the additional disequality assumptions but assumes that $x^{2}=y^{2}$. Since we are considering the asymmetric case, the only option is $x=-y$. By assumption of Case 2, we have that $w^{3} \neq-z^{3}$. Under these conditions, the projectors in row 2 of Table 1 have the desired properties.

Like Cases 1 and 2, Case 3 retains the assumption that no variable is zero but now considers the case that the polynomial $w^{3} x+w x y z+w^{2} z^{2}+y z^{3}$ is zero. Given that we are also considering the asymmetric case (i.e., $x \neq y$ ), the projectors in row 3 of Table 1 have the desired properties.

Cases 4 and 5 handle the remaining case of $(w z \neq x y) \wedge(w x y z=0)$. The assumptions $[w z \neq$ $x y] \wedge[(w, z) \neq(0,0)] \wedge[(x, y) \neq(0,0)]$ imply that at most one of $w, x, y$, and $z$ is 0 and, together with $w x y z=0$, exactly one of $w, x, y$, and $z$ is 0 . By switching the role of 0 and 1 via the holographic transformation $\left[\begin{array}{ll}0 & 1 \\ 1 & 0\end{array}\right]$, the complexity of the case $y z=0$ is the same as the complexity of the case $w x=0$. Therefore, we may assume that $y z \neq 0$. Case 4 considers $w=0$. We also have that $z \neq 0$. Then, still within the asymmetric case, the projectors in row 4 of Table 1 have the desired properties. Case 5 considers $x=0$. We also have that $y \neq 0$. Then, the projectors in row 5 of Table 1 have the desired properties.

These five cases cover all settings not excluded by the assumptions in the statement of the lemma; thus, the proof is complete.

Once we have all unary signatures at our disposal, we can prove \#P-hardness under most settings.

Lemma 4.9. Let $w, x, y, z \in \mathbb{C}$ and let $\mathcal{G}$ and $\mathcal{R}$ be finite signature sets with $(w, x, y, z) \in \mathcal{G}$ and $\left(==_{3}\right) \in \mathcal{R}$. Suppose that Holant $\left(\mathcal{G} \cup\left\{\left(X_{i}, Y_{i}\right) \mid 0 \leq i<m\right\} \mid \mathcal{R}\right) \leq_{T} \operatorname{Holant}(\mathcal{G} \mid \mathcal{R})$ for any $X_{i}, Y_{i} \in \mathbb{C}$ and $m \in \mathbb{Z}^{+}$. Then, Holant $(\mathcal{G} \mid \mathcal{R})$ is $\# \mathrm{P}$-hard unless

$$
[w z=x y] \vee[(w, z)=(0,0)] \vee[(x, y)=(0,0)] .
$$

The proof of Lemma 4.9 makes use of the following lemma.

Lemma 4.10 (Lemma 3.3 of [35] (see Also [36])). Suppose that $(a, b) \in \mathbb{C}^{2}-\{(a, b) \mid a b=1\}-$ $\{(0,0)\}$ and let $\mathcal{G}$ and $\mathcal{R}$ be finite signature sets, where $(a, 1,1, b) \in \mathcal{G}$ and $\left(=_{3}\right) \in \mathcal{R}$. Further assume that Holant $\left.\left(\mathcal{G} \cup\left\{\left(X_{i}, Y_{i}\right)\right\} \mid 0 \leq i<m\right\} \mid \mathcal{R}\right) \leq_{T} \operatorname{Holant}(\mathcal{G} \mid \mathcal{R})$ for any $X_{i}, Y_{i} \in \mathbb{C}$ and $m \in \mathbb{Z}^{+}$. Then, $\operatorname{Holant}(\mathcal{G} \cup\{(0,1,1,1)\} \mid \mathcal{R}) \leq_{T} \operatorname{Holant}(\mathcal{G} \mid \mathcal{R})$ and $\operatorname{Holant}(\mathcal{G} \mid \mathcal{R})$ is \#P-hard.

Proof of Lemma 4.9. We show that Holant $\left((0,1,1,1) \mid==_{3}\right)$, the \#P-complete problem of counting vertex covers on 3-regular graphs, can be reduced to $\operatorname{Holant}(\mathcal{G} \mid \mathcal{R})$. Since $\left(=_{3}\right) \in \mathcal{R}$, we need only to show how to simulate the generator signature $(0,1,1,1)$. We assume that $[w z \neq$ $x y] \wedge[(w, z) \neq(0,0)] \wedge[(x, y) \neq(0,0)]$. Then, at most one of $w, x, y$, and $z$ is zero. By switching the role of 0 and 1 via the holographic transformation $\left[\begin{array}{ll}0 & 1 \\ 1 & 0\end{array}\right]$, the complexity of the case $y z=0$ is the same as the complexity of the case $w x=0$. Therefore, we assume that $y z \neq 0$.

If $w=0$, then Gadget 7 using the unary $\theta=\frac{1}{x}\left(\frac{z}{y^{2}}, \frac{1}{z}\right)$ has signature

$$
\left[\begin{array}{ll}
0 & x \\
y & z
\end{array}\right]\left[\begin{array}{cc}
\frac{z}{x y^{2}} & 0 \\
0 & \frac{1}{x z}
\end{array}\right]\left[\begin{array}{ll}
0 & y \\
x & z
\end{array}\right]=\left[\begin{array}{cc}
\frac{x}{z} & 1 \\
1 & \frac{2 z}{x}
\end{array}\right],
$$




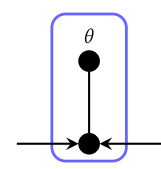

Gadget 7

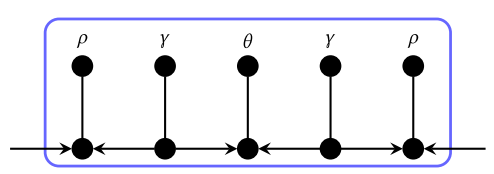

Gadget 8

Fig. 7. Gadgets used to simulate the generator $(0,1,1,1)$.

which is the binary signature $\left(\frac{x}{z}, 1,1, \frac{2 z}{x}\right)$. This signature, in turn, can simulate $(0,1,1,1)$ by Lemma 4.10. If $x=0$, then Gadget 7 using the unary $\theta=\frac{1}{w}\left(\frac{1}{y}, \frac{y}{z^{2}}\right)$ has signature

$$
\left[\begin{array}{ll}
w & 0 \\
y & z
\end{array}\right]\left[\begin{array}{cc}
\frac{1}{w y} & 0 \\
0 & \frac{y}{w z^{2}}
\end{array}\right]\left[\begin{array}{cc}
w & y \\
0 & z
\end{array}\right]=\left[\begin{array}{cc}
\frac{w}{y} & 1 \\
1 & \frac{2 y}{w}
\end{array}\right],
$$

which is the binary signature $\left(\frac{w}{y}, 1,1, \frac{2 y}{w}\right)$. This signature, in turn, can simulate $(0,1,1,1)$ by Lemma 4.10. If $[w x \neq 0] \wedge[w z=-x y]$, then Gadget 7 with $\theta=\frac{1}{x y}\left(\frac{2 x}{w}, \frac{w}{x}\right)$ gives the binary signature $\left(\frac{3 w}{y}, 1,1, \frac{3 y}{w}\right)$, which, in turn, can simulate $(0,1,1,1)$ by Lemma 4.10 .

Finally, if $[w x \neq 0] \wedge[w z \neq x y] \wedge[w z \neq-x y]$, then Gadget 8 with

$$
\begin{aligned}
& \theta=\frac{w z+x y}{w x(w z-x y)}\left(\frac{-x}{w}, \frac{w}{x}\right), \\
& \gamma=\left(\frac{1}{w x}, \frac{-w x}{y z(w z+x y)}\right), \text { and } \\
& \rho=\frac{1}{w z-x y}(x z,-w y)
\end{aligned}
$$

has the signature $(0,1,1,1)$.

By combining the Group Lemma (Lemma 4.6) with Lemma 4.8 and Lemma 4.9, we get the following theorem.

Theorem 4.11. Let $w, x, y, z \in \mathbb{C}$ and let $\mathcal{G}$ and $\mathcal{R}$ be finite signature sets, where $(w, x, y, z) \in \mathcal{G}$ and $\left(=_{3}\right) \in \mathcal{R}$. Suppose that $\mathcal{S}$ is a finite set of recursive $(\mathcal{G} \mid \mathcal{R})$-gates of arity $d \geq 1$ with nonsingular transition matrices. Let $H$ be the group generated by the transition matrices of $\mathcal{S}$, modulo scalar matrices $\lambda I$, for $\lambda \in \mathbb{C}-\{0\}$. If $H$ has infinite order, then Holant $(\mathcal{G} \mid \mathcal{R})$ is \#P-hard unless

$$
[x=y] \vee[w z=x y] \vee[(w, z)=(0,0)] \vee[(x, y)=(0,0)] \vee\left[\left(w^{3}=-z^{3}\right) \wedge(x=-y)\right] .
$$

Proof. For any signature $f=(w, x, y, z)$ satisfying the hypothesis, by Lemma 4.8 there is a finite projective $\left(f \mid==_{3}\right)$-gate set from arity 2-to-1. Then, the conditions of the Group Lemma (Lemma 4.6) are satisfied, thus, we can get any unary signature $(X, Y)$. Iterating this any constant number of times will satisfy the conditions of Lemma 4.9, which proves that $\operatorname{Holant}(\mathcal{G} \mid \mathcal{R})$ is \#P-hard.

We remark that the exceptions listed in Theorem 4.11 are different than that listed for Lemma 4.9; in Lemma 4.9, all unary signatures are assumed, while in Theorem 4.11 they are obtained by Lemma 4.6 and Lemma 4.8.

\section{MAIN RESULT}

Theorem 5.1. Let $w, x, y, z \in \mathbb{C}$ and $f=(w, x, y, z)$. Then, Holant $\left(f \mid={ }_{3}\right)$ is \#P-hard except in the following classes, for which the problem is computable in polynomial time: 
(1) degenerate: $w z=x y$;

(2) generalized disequality: $w=z=0$;

(3) generalized equality: $x=y=0$;

(4) affine after holographic transformation: $[w z=-x y] \wedge\left[w^{6}=\varepsilon z^{6}\right] \wedge\left[x^{2}=\varepsilon y^{2}\right]$, where $\varepsilon= \pm 1$

If the input is restricted to planar graphs, then another class becomes computable in polynomial time but everything else remains \#P-hard:

(5) holographic algorithms with matchgates: $\left[w^{3}=\varepsilon z^{3}\right] \wedge[x=\varepsilon y]$, where $\varepsilon= \pm 1$.

We remark that when restricted to symmetric signatures, that is, $x=y$, the dichotomy has already been proved in [35] (see also [36]), and the statement is indeed the special case of Theorem 5.1. We first give a simple lemma that demystifies the explicit description of an important tractable case in Theorem 5.1, the affine signatures. However, this lemma is not logically required for the proof of Theorem 5.1.

LemmA 5.2. For $x_{1}, x_{2} \in\{0,1\}$, let $f\left(x_{1}, x_{2}\right)=(1, X, Y, Z)$. Then $[Z=-X Y] \wedge\left[Z^{2}=\varepsilon\right] \wedge\left[X^{2}=\right.$ $\left.\varepsilon Y^{2}\right]$ with $\varepsilon= \pm 1$ iff there exist $b, c \in \mathbb{Z} / 4 \mathbb{Z}$ such that

$$
f\left(x_{1}, x_{2}\right)=\mathfrak{i}^{2 x_{1} x_{2}+b x_{1}+c x_{2}} .
$$

Proof. The proof is by a direct verification on the 16 possible cases, eight of which have $\varepsilon=1$, which correspond to $b+c \equiv 0(\bmod 2)$, while the other eight cases have $\varepsilon=-1$, which correspond to $b+c \equiv 1(\bmod 2)$.

We prove the tractability part of Theorem 5.1 next. The proof of \#P-hardness is presented in Section 6.

Proof of Tractability. For any signature grid $\Omega$, Holant $\Omega$ is the product of the Holant on each connected component. Case 1 is degenerate. We can break up every edge into two unary functions; then, the Holant value is a simple product over all vertices. For case 2, on any connected component, the Holant value is zero unless it is bipartite and, if so, a 2-coloring algorithm can be used to find the only two assignments that can have a nonzero value, which are complements of each other. Similarly, for case 3, only the all- 0 and all-1 assignments can possibly yield a nonzero value for each connected component. For case 4 , if $w=0$ or $z=0$, then by $w^{3}=\varepsilon z^{3}$, they are both zero, which is already covered by case 2 . Now, suppose that $w z \neq 0$. Since $w^{6}=\varepsilon z^{6}$, we can apply the holographic transformation $\left[\begin{array}{cc}\alpha & 0 \\ 0 & \alpha^{2}\end{array}\right]$ with $\alpha=\varepsilon w^{2} / z^{2}$. Note that $\alpha^{3}=\varepsilon w^{6} / z^{6}=1$. The edge signature becomes $(w, x, y, z)\left[\begin{array}{cc}\alpha & 0 \\ 0 & \alpha^{2}\end{array}\right]^{\otimes 2}=\left(\alpha^{2} w, x, y, \alpha z\right)$, which we denote by $(W, X, Y, Z)$, while $(=3)$ is unchanged since $\left[\begin{array}{cc}\alpha & 0 \\ 0 & \alpha^{2}\end{array}\right]^{-1}=\left[\begin{array}{cc}\alpha^{2} & 0 \\ 0 & \alpha\end{array}\right]$, and

$$
\left[\begin{array}{cc}
\alpha^{2} & 0 \\
0 & \alpha
\end{array}\right]^{\otimes 3}\left\{\left[\begin{array}{l}
1 \\
0
\end{array}\right]^{\otimes 3}+\left[\begin{array}{l}
0 \\
1
\end{array}\right]^{\otimes 3}\right\}=\left[\begin{array}{c}
\alpha^{2} \\
0
\end{array}\right]^{\otimes 3}+\left[\begin{array}{l}
0 \\
\alpha
\end{array}\right]^{\otimes 3}=\left[\begin{array}{l}
1 \\
0
\end{array}\right]^{\otimes 3}+\left[\begin{array}{l}
0 \\
1
\end{array}\right]^{\otimes 3} .
$$

This becomes equivalent to the case $[W Z=-X Y] \wedge\left[W^{2}=\varepsilon Z^{2}\right] \wedge\left[X^{2}=\varepsilon Y^{2}\right]$, where $\varepsilon= \pm 1$. This edge signature $(W, X, Y, Z)$ belongs to the so-called affine function family and is tractable by Theorem 5.2 of [17]. For case 5, if the input is restricted to planar graphs, then we use the theory of holographic algorithms with matchgates to compute the Holant in polynomial time (see [16, 41]). 
We give an alternative and more conceptual description of all tractable cases in Theorem 5.1. Case 1 is degenerate: $\operatorname{det}\left[\begin{array}{ll}f(0,0) & f(0,1) \\ f(1,0) & f(1,1)\end{array}\right]=0$. Case 2 is generalized disequality: $\left[\begin{array}{cc}0 & f(0,1) \\ f(1,0) & 0\end{array}\right]$. Case 3 is generalized equality: $\left[\begin{array}{cc}f(0,0) & 0 \\ 0 & f(1,1)\end{array}\right]$.

Case 4 is more interesting: For $f=\left[\begin{array}{ll}w & x \\ y & z\end{array}\right]$, we have that $[w z=-x y] \wedge\left[w^{6}=\varepsilon z^{6}\right] \wedge\left[x^{2}=\varepsilon y^{2}\right]$, where $\varepsilon= \pm 1$. If any of $w, x, y, z=0$, then the above condition forces all $w=x=y=z=0$. This constant 0 function is trivially tractable. Assume that $w x y z \neq 0$. We let $(W, X, Y, Z)=$ $\left(\alpha^{2} w, x, y, \alpha z\right)$, where $\alpha=\varepsilon w^{2} / z^{2}$. Thus, $W=w^{5} / z^{4}$. In the proof of tractability of Theorem 5.1, it is shown that under a holographic transformation, this is equivalent to $[W Z=-X Y] \wedge\left[W^{2}=\right.$ $\left.\varepsilon Z^{2}\right] \wedge\left[X^{2}=\varepsilon Y^{2}\right]$, where $\varepsilon= \pm 1$. If we further normalize $f$ by setting $W=1$, which corresponds to a global nonzero constant factor, then this tractable case is equivalent to $[Z=-X Y] \wedge\left[Z^{2}=\right.$ $\varepsilon] \wedge\left[X^{2}=\varepsilon Y^{2}\right]$, where $\varepsilon= \pm 1$. This has a simple form as an exponential quadratic polynomial by Lemma 5.2.

Without normalizing $W=1$, and including some degenerate cases, we can use

$$
f\left(x_{1}, x_{2}\right)=\lambda \mathfrak{i}^{2 a x_{1} x_{2}+b x_{1}+c x_{2}},
$$

where $\lambda \in \mathbb{C}$ contributes only a constant factor and the case $a=0$ is degenerate.

\section{ANTI-GADGETS IN ACTION}

In this section, we give the \#P-hardness proof in Theorem 5.1. A main step is to use anti-gadgets to explicitly construct gadgets with signature matrices having infinite order.

Lemma 6.1. If $w z \neq x y, w x y z \neq 0$, and $|x| \neq|y|$, then Holant $\left((w, x, y, z) \mid==_{3}\right)$ is \#P-hard.

Proof. As described in Section 3, the transition matrices for Gadget 4 and Gadget 5 are $M_{4}=\left[\begin{array}{ll}w & x \\ y & z\end{array}\right]^{\otimes 2} \operatorname{diag}(w, x, y, z)$ and $M_{5}=\left[\begin{array}{ll}w & x \\ y & z\end{array}\right]^{\otimes 2} \operatorname{diag}(w, y, x, z)$, respectively. Both are nonsingular. Since the matrix $M_{4}^{-1} M_{5}=\operatorname{diag}(1, y / x, x / y, 1)$ has infinite order up to a scalar, we are done by Theorem 4.11.

Lemma 6.2. If $x=0$ and $w y z \neq 0$, then $\operatorname{Holant}\left((w, x, y, z) \mid={ }_{3}\right)$ is $\# \mathrm{P}-$ hard.

Proof. The transition matrices for Gadget 2 and Gadget 3 are $M_{2}=\left[\begin{array}{cc}w & 0 \\ y & z\end{array}\right]\left[\begin{array}{cc}w^{2} & y^{2} \\ 0 & z^{2}\end{array}\right]$ and $M_{3}=$ $\left[\begin{array}{ll}w & 0 \\ y & z\end{array}\right]\left[\begin{array}{cc}w^{2} & 0 \\ 0 & z^{2}\end{array}\right]$, both nonsingular. Since $M_{3}^{-1} M_{2}=\left[\begin{array}{cc}1 & y^{2} / w^{2} \\ 0 & 1\end{array}\right]$ has infinite order up to a scalar, we are done by Theorem 4.11 .

Lemma 6.3. If $w=0$ and $x y z \neq 0$, then $\operatorname{Holant}\left((w, x, y, z) \mid={ }_{3}\right)$ is $\# \mathrm{P}-$ hard.

Proof. The transition matrices for Gadget 3 and Gadget 6 are $M_{3}=\left[\begin{array}{ll}0 & x \\ y & z\end{array}\right]\left[\begin{array}{cc}0 & x y \\ x y & z^{2}\end{array}\right]$ and $M_{6}=$ $\left[\begin{array}{ll}0 & x \\ y & z\end{array}\right]\left[\begin{array}{cc}0 & x y z^{3} \\ x y z^{3} & x y^{2} z^{2}+z^{5}\end{array}\right]$, both nonsingular. Since $M_{3}^{-1} M_{6}=z^{3}\left[\begin{array}{cc}1 & y / z \\ 0 & 1\end{array}\right]$ has infinite order up to a scalar, we are done by Theorem 4.11 .

For the remainder of the proof of \#P-hardness of Theorem 5.1, we use our anti-gadget technique in combination with Lemma 6.4, Lemma 6.5, and Lemma 6.6. In the contrapositive, these lemmas provide sufficient conditions to conclude that a matrix has infinite order (up to a scalar). Their proofs follow from a few observations. For monic polynomials in $\mathbb{C}[X]$ of degree $n$ with roots $\lambda_{i}$ for $1 \leq i \leq n$ of the same nonnegative norm $r \in \mathbb{R}$, let $a_{k} \in \mathbb{C}$ be the coefficient of $X^{k}$ and let $\sigma_{k}$ be the elementary symmetric polynomial of degree $k$ in $\lambda_{i} / r$ for $1 \leq i \leq n$, the norm one (scaled) roots. ${ }^{2}$

\footnotetext{
${ }^{2}$ This argument assumes that $r \neq 0$. However, when $r=0$, the conclusion still holds trivially.
} 
Thus, $a_{k}=(-r)^{n-k} \sigma_{n-k}$. Now, the numbers $\lambda_{i} / r$ are of norm one and we have that $\sigma_{k}=\overline{\sigma_{n-k}} \sigma_{n}$, $\left|\sigma_{n}\right|=1$; thus, $a_{k}=(-1)^{n} r^{n-2 k} \overline{a_{n-k}} \sigma_{n}$ and $\left|a_{k}\right|=r^{n-2 k}\left|a_{n-k}\right|$ for $0 \leq k<n$.

Lemma 6.4 (Lemma 4.4 in [35] (see Also [36])). If both roots of $X^{2}+a_{1} X+a_{0} \in \mathbb{C}[X]$ have the same norm, then $a_{1}\left|a_{0}\right|=\overline{a_{1}} a_{0}$. If further $a_{0} a_{1} \neq 0$, then $\operatorname{Arg}\left(a_{1}^{2}\right)=\operatorname{Arg}\left(a_{0}\right)$; thus, $a_{1}^{2} / a_{0} \in \mathbb{R}^{+}$, the positive real numbers.

LemmA 6.5. If all roots of $X^{4}+a_{3} X^{3}+a_{2} X^{2}+a_{1} X+a_{0} \in \mathbb{C}[X]$ have the same norm, then $a_{2}\left|a_{1}\right|^{2}=\left|a_{3}\right|^{2} \overline{a_{2}} a_{0}$.

Proof. The left-hand side is $r^{2} \sigma_{2} r^{6}\left|\sigma_{3}\right|^{2}$, while the right-hand side is $r^{2}\left|\sigma_{1}\right|^{2} r^{2} \overline{\sigma_{2}} r^{4} \sigma_{4}$. Substituting $\sigma_{2}=\overline{\sigma_{2}} \sigma_{4}$ and $\sigma_{3}=\overline{\sigma_{1}} \sigma_{4}$, and using $\left|\sigma_{4}\right|=1$, the equality follows.

Lemma 6.6. If $\sum_{k=0}^{8} a_{k} X^{k} \in \mathbb{C}[X]$ is monic and all roots have the same norm, then $a_{3}^{2}\left|a_{1}\right|^{2}=$ $\left|a_{7}\right|^{2}{\overline{a_{5}}}^{2} a_{0}^{2}, a_{4}\left|a_{2}\right|^{2}=\left|a_{6}\right|^{2} \overline{a_{4}} a_{0}$, and $\left|a_{3}\right|^{2} a_{2}=\overline{a_{6}}\left|a_{5}\right|^{2} a_{0}$.

Proof. For the equality $a_{3}^{2}\left|a_{1}\right|^{2}=\left|a_{7}\right|^{2}{\overline{a_{5}}}^{2} a_{0}^{2}$, the left-hand side is $r^{10} \sigma_{5}^{2} r^{14}\left|\sigma_{7}\right|^{2}$, while the righthand side is $r^{2}\left|\sigma_{1}\right|^{2} r^{6}{\overline{\sigma_{3}}}^{2} r^{16} \sigma_{8}^{2}$. Substituting $\sigma_{5}=\overline{\sigma_{3}} \sigma_{8}, \sigma_{7}=\overline{\sigma_{1}} \sigma_{8}$, and using $\left|\sigma_{8}\right|=1$, the equality follows. The other two equalities are similarly proved.

On directed 3-regular graphs, there are some symmetries under which the Holant is invariant. The next lemma states these symmetries.

Lemma 6.7. Let $G$ be a directed 3-regular graph. Then there exists a polynomial $P$ with integer coefficients in six variables such that for any signature grid $\Omega$ having underlying graph $G$ with each vertex given the signature $=_{3}$ and each edge given the signature $(w, x, y, z)$, the Holant value is

$$
\text { Holant }_{\Omega}=P\left(w z, x y, w^{3}+z^{3}, x+y, w^{3} x+y z^{3}, w^{3} y+x z^{3}\right) .
$$

Proof. Consider any $\{0,1\}$-vertex assignment $\sigma$ with a nonzero valuation. If $\sigma^{\prime}$ is the complement assignment switching all 0 s and $1 \mathrm{~s}$ in $\sigma$, then for $\sigma$ and $\sigma^{\prime}$, we have the sum of valuations $w^{a} x^{b} y^{c} z^{d}+w^{d} x^{c} y^{b} z^{a}$ for some $a, b, c, d$. Here, $a$ (resp., $d$ ) is the number of edges connecting two degree 3 vertices both assigned 0 (resp., 1) by $\sigma$. Similarly, $b$ (resp., $c$ ) is the number of edges from one degree 3 vertex to another that are assigned 0 and 1 (resp., 1 and 0 ), in that order, by $\sigma$. We note that

$$
\begin{aligned}
& w^{a} x^{b} y^{c} z^{d}+w^{d} x^{c} y^{b} z^{a} \\
& \quad=\left\{\begin{array}{l}
(w z)^{\min (a, d)}(x y)^{\min (b, c)}\left(w^{|a-d|} y^{|b-c|}+x^{|b-c|} z^{|a-d|}\right) a>d \text { XOR } b>c \\
(w z)^{\min (a, d)}(x y)^{\min (b, c)}\left(w^{|a-d|} x^{|b-c|}+y^{|b-c|} z^{|a-d|}\right) \text { otherwise. }
\end{array}\right.
\end{aligned}
$$

We prove $a \equiv d(\bmod 3)$ inductively. For the all-0 assignment, this is clear since every edge contributes a factor $w$ and the number of edges is divisible by 3 for a 3-regular graph. Now, starting from any assignment, if we switch the assignment on one vertex from 0 to 1 , it is easy to verify that it changes the valuation from $w^{a} x^{b} y^{c} z^{d}$ to $w^{a^{\prime}} x^{b^{\prime}} y^{c^{\prime}} z^{d^{\prime}}$, where $a-d=a^{\prime}-d^{\prime}+3$. As every $\{0,1\}$-assignment is obtainable from the all-0 assignment by a sequence of switches, the conclusion $a \equiv d(\bmod 3)$ follows.

Now,

$$
\begin{aligned}
& w^{a} x^{b} y^{c} z^{d}+w^{d} x^{c} y^{b} z^{a} \\
& =\left\{\begin{array}{l}
(w z)^{\min (a, d)}(x y)^{\min (b, c)}\left(w^{3 k} y^{\ell}+x^{\ell} z^{3 k}\right) a>d \text { XOR } b>c \\
(w z)^{\min (a, d)}(x y)^{\min (b, c)}\left(w^{3 k} x^{\ell}+y^{\ell} z^{3 k}\right) \text { otherwise }
\end{array}\right.
\end{aligned}
$$


for some $k, \ell \geq 0$. Consider $w^{3 k} y^{\ell}+x^{\ell} z^{3 k}$ (the other case is similar). Two simple inductive steps

$$
\begin{aligned}
w^{3 k} y^{\ell+1}+x^{\ell+1} z^{3 k} & =\left(w^{3 k} y^{\ell}+x^{\ell} z^{3 k}\right)(x+y)-x y\left(w^{3 k} y^{\ell-1}+x^{\ell-1} z^{3 k}\right) \\
w^{3(k+1)} y^{\ell}+x^{\ell} z^{3(k+1)} & =\left(w^{3 k} y^{\ell}+x^{\ell} z^{3 k}\right)\left(w^{3}+z^{3}\right)-(w z)^{3}\left(w^{3(k-1)} y^{\ell}+x^{\ell} z^{3(k-1)}\right)
\end{aligned}
$$

(when combined with the other case) show that the Holant is indeed a polynomial $P\left(w z, x y, w^{3}+\right.$ $\left.z^{3}, x+y, w^{3} x+y z^{3}, w^{3} y+x z^{3}\right)$ with integer coefficients.

Assuming nondegeneracy of $(w, x, y, z)$, Lemma 6.1, Lemma 6.2, and Lemma 6.3 give \#Phardness unless two (or more) of $w, x, y$, and $z$ are zero or none are zero and $|x|=|y|$. If any two (or more) of variables are zero, then the problem is tractable, as proved in the tractability proof for Theorem 5.1. Therefore, the dichotomy in Theorem 5.1 holds unless $w x y z \neq 0$ and $|x|=|y|$. In accordance with Lemma 6.7, we make a change of variables to

$$
A=w z, \quad B=x y, \quad C=w^{3}+z^{3}, \quad D=x+y, \quad E=w^{3} x+y z^{3}, \quad F=w^{3} y+x z^{3} .
$$

Since the complexity of a Holant remains the same under multiplication by a nonzero constant to any signature, and $|x|=|y| \neq 0$, we may normalize so that $|x|=1$ and $x=\bar{y}$. In the following, we make this normalization. Thus, $B=1$ and $D=x+y \in[-2,2]$ with $D^{2}=4$ corresponding to the symmetric case: $x=y$. A degenerate edge signature now means $A=1$. Additionally, note that $E+F=C D$ and $E F=-4 A^{3} B+B C^{2}+A^{3} D^{2}$. Theorem 5.1 can also be stated in these symmetrized variables.

Theorem 6.8. Suppose that $w, x, y, z \in \mathbb{C}$. Then, Holant $\left((w, x, y, z) \mid={ }_{3}\right)$ is $\# \mathrm{P}$-hard except in the following cases, for which the problem is computable in polynomial time:

(1) $w z=x y \Longleftrightarrow A=B$;

(2) $w=z=0 \Longleftrightarrow A=C=0$;

(3) $x=y=0 \Longleftrightarrow B=D=0$;

(4) $w z=-x y \wedge w^{6}=z^{6} \wedge x^{2}=y^{2} \Longleftrightarrow A=-B \wedge 4 A^{3} C=C^{3} \wedge 4 B D=D^{3}$;

(5) $w z=-x y \wedge w^{6}=-z^{6} \wedge x^{2}=-y^{2} \Longleftrightarrow A=-B \wedge 2 A^{3}=C^{2} \wedge 2 B=D^{2}$.

If the input is restricted to planar graphs, then two more cases become tractable but all other cases remain \#P-hard:

(6) $w^{3}=z^{3} \wedge x=y \Longleftrightarrow 4 A^{3}=C^{2} \wedge 4 B=D^{2}$;

(7) $w^{3}=-z^{3} \wedge x=-y \Longleftrightarrow C=D=0$.

Now, we continue with the proof of \#P-hardness.

Lemma 6.9. If $D^{2} \neq 4$, and $A \notin \mathbb{R}$, then $\operatorname{Holant}\left((w, x, y, z) \mid={ }_{3}\right)$ is \#P-hard.

Proof. The transition matrices for Gadget 15 and Gadget 16 are

$$
\begin{aligned}
M_{15} & =\left[\begin{array}{ll}
w & y \\
x & z
\end{array}\right]\left[\begin{array}{ll}
w^{2} & x y \\
x y & z^{2}
\end{array}\right] \text { and } \\
M_{16} & =\left[\begin{array}{ll}
w & x \\
y & z
\end{array}\right]\left[\begin{array}{ll}
w^{2} & x y \\
x y & z^{2}
\end{array}\right] .
\end{aligned}
$$

Both matrices have determinant $(A-1)^{2}(A+1)$, which is nonzero since $A$ is not real. Then, $N=$ $M_{15} M_{16}^{-1}$ has determinant 1 and trace

$$
\operatorname{tr}\left(\left[\begin{array}{ll}
w & y \\
x & z
\end{array}\right]\left[\begin{array}{ll}
w & x \\
y & z
\end{array}\right]^{-1}\right)=\frac{2 w z-x^{2}-y^{2}}{w z-x y}=\frac{2 A-D^{2}+2}{A-1}
$$


which is nonzero since $A$ is not real. If the eigenvalues of $N$ have distinct norms, then it has infinite order up to a scalar and we are done by Theorem 4.11; thus, assume that its eigenvalues are of equal norm. Then, Lemma 6.4 says that $\frac{\operatorname{tr}(N)^{2}}{\operatorname{det} N}=\frac{\left(2 A-D^{2}+2\right)^{2}}{(A-1)^{2}} \in \mathbb{R}^{+}$. Taking square roots, we have that $\frac{2 A-D^{2}+2}{A-1} \in \mathbb{R}$, which implies that $\frac{-D^{2}+4}{A-1} \in \mathbb{R}$. Since $D^{2} \neq 4$, this gives $A \in \mathbb{R}$, a contradiction.

The case $A=1$ is degenerate (thus tractable), the case $A=0$ is covered in Lemma 6.3, and recall that $D^{2}=4$ corresponds to the symmetric case that was proved in $[35,36]$. Thus, now, we assume that $[A \neq 0,1] \wedge D^{2} \neq 4$. First, suppose that $A \neq-1$. Lemma 6.10 below handles the case $A \neq-1$ and $D \neq 0$. Then, Lemma 6.11 handles the case $A \neq-1 \wedge D=0 \wedge C \neq 0$. The case $D=0 \wedge C=0$ will be discussed later. We note that $C=D=0$ is tractable over planar graphs. After Lemma 6.10 and Lemma 6.11, we focus on the case $A=-1$.

Unary recursive gadgets, such as the ones used in the proof of Lemma 6.9, are quite useful for proving \#P-hardness when variables such as $A=w z$ are complex. When all variables are real, the conclusion of Lemma 6.4 is weak (though one can still prove \#P-hardness using a related lemma with significant effort in the symmetric case [19]). For complex variables in the symmetric case, $[35,36]$ showed that using higher arity (i.e., binary) recursive gadgets can give a much simpler proof of \#P-hardness. The next lemma continues this pattern with the first ever use of ternary recursive gadgets.

LemmA 6.10. If $A^{2} \neq 1, A D \neq 0$, and $D^{2} \neq 4$, then $\operatorname{Holant}\left((w, x, y, z) \mid={ }_{3}\right)$ is $\# \mathrm{P}$-hard

Proof. The determinants of the 8-by-8 transition matrices of Gadget 26 and Gadget 27 are both $A^{4}(A-1)^{16} \neq 0$. If $M_{26}$ has infinite order up to a scalar, then we are done by Theorem 4.11. Otherwise, we construct an anti-gadget with signature matrix $M_{26}^{-1}$ and combine it with Gadget 27 to construct a gadget with signature matrix $N=M_{26}^{-1} M_{27}$, which has determinant 1 . If $N$ has any two eigenvalues with distinct norms, then it has infinite order up to a scalar and we are done by Theorem 4.11. Thus, assume that all eight eigenvalues of $N$ have the same norm. Then, by Lemma 6.6, we know that the three equations in the statement of Lemma 6.6 hold among the coefficients of its characteristic polynomial. After scaling by the nonzero factor $A(A-1)$, these coefficients for $A(A-1) N$ are

$$
\begin{aligned}
& a_{7}=(A-1)\left(A D^{2}+2 A+2\right) \\
& a_{6}=(A-1)^{2}\left(5 A^{2} D^{2}-3 A^{2}+2 A D^{2}+2 A+1\right) \\
& a_{5}=A(A-1)^{3}\left(A^{2} D^{4}+5 A^{2} D^{2}-6 A^{2}+7 A D^{2}-6 A+D^{2}\right) \\
& a_{4}=A^{2}(A-1)^{4}\left(3 A^{2} D^{4}-4 A^{2} D^{2}+4 A^{2}+A D^{4}+4 A D^{2}-4 A+2 D^{2}-2\right) \\
& a_{3}=A^{3}(A-1)^{5}\left(2 A D^{4}+3 A^{2} D^{4}-6 A^{2} D^{2}+6 A^{2}-4 A D^{2}+6 A+D^{2}\right) \\
& a_{2}=A^{4}(A-1)^{6}\left(A^{2} D^{4}+A^{2} D^{2}-3 A^{2}+A D^{4}-2 A D^{2}+2 A+1\right) \\
& a_{1}=A^{6}(A-1)^{7}\left(2 A D^{2}-2 A+D^{2}-2\right) \\
& a_{0}=A^{8}(A-1)^{8} .
\end{aligned}
$$

Amazingly, $C, E$, and $F$ do not appear. Lemma 6.9 shows \#P-hardness unless $A \in \mathbb{R}$; thus, assume that $A \in \mathbb{R}$. Recall that $D \in[-2,2]$; we have that both $A, D \in \mathbb{R}$, the equations in Lemma 6.6 are simplified by the disappearance of norms and conjugates. Using CylindricalDecomposiTION in Mathematica, we conclude that there are no solutions under our assumptions, which is a contradiction. ${ }^{3}$

\footnotetext{
${ }^{3}$ The fact that $C, E$, and $F$ do not appear helps. The runtime of CylindricalDecomposition is double exponential in the number of variables in the worst case; thus, it is crucial that our query include as few variables as possible.
} 
We remark that the use of CyLINDRICALDECOMPosition in the proof of Lemma 6.10 is the only essential use of Mathematica in this article. All other uses of Mathematica can be verified by hand. If implemented properly, CYLINDRICALDECOMPOSITION provides a mathematical proof that no solution exists, not merely that it could not find a solution.

Since we assumed that $A \neq 0,1$, the meaning of the assumptions in Lemma 6.10 is simply that $A \neq-1$ and $D \neq 0$ in the nonsymmetric case $(x \neq y)$. The next lemma considers the same assumptions except that $D$ is zero and $C$ is nonzero.

Lemma 6.11. If $A^{2} \neq 1, A C \neq 0$, and $D=0$, then $\operatorname{Holant}\left((w, x, y, z) \mid={ }_{3}\right)$ is $\# \mathrm{P}$-hard.

Proof. Lemma 6.9 shows \#P-hardness unless $A \in \mathbb{R}$; thus, assume that $A \in \mathbb{R}$. The transition matrix for Gadget 19 is $M_{19}=\left[\begin{array}{ll}w & x \\ y & z\end{array}\right]^{\otimes 2} \operatorname{diag}(w, x, y, z)$ and has determinant $A(A-1)^{4} \neq 0$. If $M_{19}$ has any two eigenvalues with distinct norms, then it has infinite order up to a scalar and we are done by Theorem 4.11; thus, assume that all eigenvalues have the same norm. However, the coefficients of the characteristic polynomial of $M_{19}$, which are

$$
\left(a_{3}, a_{2}, a_{1}, a_{0}\right)=\left(-C,(A+1)^{2}(A-1),-(A-1)^{2} C, A(A-1)^{4}\right),
$$

do not satisfy the conclusion of Lemma 6.5 under the assumptions, a contradiction.

We now focus on the case $A=-1$. The \#P-hardness proofs of Lemma 6.13 and Lemma 6.14 make use of the following technical lemma.

Lemma 6.12. Let $c \in \mathbb{C}$ and $\varepsilon= \pm 1$. Then, the only solutions to the equation $(\overline{c+2 \varepsilon}) c=\varepsilon(c+2 \varepsilon)$ are the trivial solutions $c \in\{-2 \varepsilon, \varepsilon\}$.

Proof. Assume that $c \neq-2 \varepsilon$. Now, we show that $c=\varepsilon$. Taking norms, we see that $|c|=1$. Then, simplifying $(\overline{c+2 \varepsilon}) c=\varepsilon(c+2 \varepsilon)$ using $c \bar{c}=|c|^{2}=1$ yields $c=\varepsilon$ as claimed.

Lemma 6.13. If $A=-1$ and $E \notin\{0, \pm 2 \mathfrak{i}\}$, then $\operatorname{Holant}\left((w, x, y, z) \mid={ }_{3}\right)$ is $\# \mathrm{P}$-hard.

Proof. The transition matrices of Gadget 21, Gadget 22, and Gadget 24 are

$$
\begin{aligned}
& M_{21}=\left[\begin{array}{ll}
w & x \\
y & z
\end{array}\right]^{\otimes 2} \operatorname{diag}(w, x, y, z)\left[\begin{array}{cc}
w & y \\
x & z
\end{array}\right]^{\otimes 2} \operatorname{diag}(w, y, x, z) \\
& M_{22}=\left[\begin{array}{ll}
w & x \\
y & z
\end{array}\right]^{\otimes 2}\left[\begin{array}{cccc}
w^{4}+w x y^{2} & w^{2} x y+x y^{2} z & 0 & 0 \\
w^{2} x y+x y^{2} z & w x y z+y z^{3} & 0 & 0 \\
0 & 0 & w^{3} x+w x y z & w x^{2} y+x y z^{2} \\
0 & 0 & w x^{2} y+x y z^{2} & x^{2} y z+z^{4}
\end{array}\right] \\
& M_{24}=\left[\begin{array}{ll}
w & x \\
y & z
\end{array}\right]^{\otimes 2} \operatorname{diag}(w, y, x, z)\left(I_{2} \otimes\left[\begin{array}{ll}
w & y \\
x & z
\end{array}\right]\left[\begin{array}{cc}
w^{2}+y z & 0 \\
0 & w x+z^{2}
\end{array}\right]\right),
\end{aligned}
$$

with $\operatorname{det} M_{21}=2^{8}, \operatorname{det} M_{22}=2^{6}\left(E^{2}+4\right)$, and $\operatorname{det} M_{24}=-2^{6} E^{2}$; thus, all are nonsingular. Let $N_{1}=$ $M_{21}^{-1} M_{22}$ and $N_{2}=M_{21}^{-1} M_{24}$. The coefficients of the characteristic polynomials of $-2^{4} N_{1}$ and $2^{4} N_{2}$ are, respectively,

$$
\begin{aligned}
& \left(a_{3}, a_{2}, a_{1}, a_{0}\right)=\left(-4, E^{2}+12,-4\left(E^{2}+4\right), 4\left(E^{2}+4\right)\right) \\
& \left(a_{3}, a_{2}, a_{1}, a_{0}\right)=\left(4,-E^{2}+8,-4 E^{2},-4 E^{2}\right) .
\end{aligned}
$$

If $M_{21}$ has infinite order up to a scalar, then we are done by Theorem 4.11. Otherwise, we can construct the anti-gadget with signature matrix $M_{21}^{-1}$ and combine it with each of Gadget 22 and Gadget 24 to construct gadgets with signature matrices $N_{1}$ and $N_{2}$, respectively. If $N_{1}$ (resp., $N_{2}$ ) has any two eigenvalues with distinct norms, then $N_{1}$ (resp., $N_{2}$ ) has infinite order up to a scalar and we 


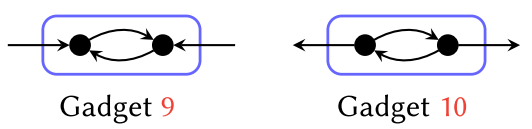

Fig. 8. Gadgets with a symmetric generator signature.

are done by Theorem 4.11. Thus, assume that all eigenvalues of $N_{1}$ (resp., $N_{2}$ ) have the same norm. Then, by Lemma 6.5, we have two equations relating these coefficients. However, after a change of variables by $c=E^{2} / 4$ (for the coefficients of $\left.N_{1}\right)$ and $c=\left(E^{2}+4\right) / 4$ (for the coefficients of $N_{2}$ ), Lemma 6.12 says that the only solutions to both equations require $E \in\{0, \pm 2 i\}$, a contradiction.

The next lemma is similar to Lemma 6.13 with $E$ in place of $F$.

LemmA 6.14. If $A=-1$ and $F \notin\{0, \pm 2 i\}$, then Holant $\left((w, x, y, z) \mid={ }_{3}\right)$ is \#P-hard.

Proof. The transition matrices of Gadget 21, Gadget 23, and Gadget 25 are

$$
\begin{aligned}
& M_{21}=\left[\begin{array}{ll}
w & x \\
y & z
\end{array}\right]^{\otimes 2} \operatorname{diag}(w, x, y, z)\left[\begin{array}{cc}
w & x \\
y & z
\end{array}\right]^{\otimes 2} \operatorname{diag}(w, y, x, z) \\
& M_{23}=\left[\begin{array}{ll}
w & x \\
y & z
\end{array}\right]^{\otimes 2}\left[\begin{array}{cccc}
w^{4}+w x^{2} y & w^{2} x y+x^{2} y z & 0 & 0 \\
w^{2} x y+x^{2} y z & w x y z+x z^{3} & 0 & 0 \\
0 & 0 & w^{3} y+w x y z & w x y^{2}+x y z^{2} \\
0 & 0 & w x y^{2}+x y z^{2} & x y^{2} z+z^{4}
\end{array}\right] \\
& M_{25}=\left[\begin{array}{ll}
w & x \\
y & z
\end{array}\right]^{\otimes 2} \operatorname{diag}(w, x, y, z)\left(I_{2} \otimes\left[\begin{array}{ll}
w & x \\
y & z
\end{array}\right]\left[\begin{array}{cc}
w^{2}+x z & 0 \\
0 & w y+z^{2}
\end{array}\right]\right) .
\end{aligned}
$$

The rest of the proof uses the same reasoning as the proof of Lemma 6.13 with Gadget 22 and Gadget 24 replaced by Gadget 23 and Gadget 25, respectively.

All remaining cases, those for which $A=-1$ and $E, F \in\{0, \pm 2 \mathfrak{i}\}$, imply tractability. Since this is not immediately obvious, we prove this next. As pointed out after Lemma 6.7, the following equations hold and are used frequently below. They simplify to

$$
\begin{aligned}
E+F & =C D \\
E F & =-4 A^{3} B+B C^{2}+A^{3} D^{2}=4+C^{2}-D^{2}
\end{aligned}
$$

when $A=-1$ and $B=1$. These next four lemmas cover all possibilities of $E, F \in\{0, \pm 2 \mathrm{i}\}$ as follows:

Lemma 6.15: Both zero;

Lemma 6.18: Exactly one zero;

Lemma 6.16: Both nonzero and equal;

Lemma 6.17: Both nonzero and not equal.

Lemma 6.15. If $A=-1 \wedge E=F=0$, then $\left(D=0 \wedge C^{2}=-4\right)$ or $\left(D^{2}=4 \wedge C=0\right)$, which are both tractable.

Proof. Since $0=E+F=C D$, either $C$ or $D$ is zero. In either case, simplifying (2) gives the desired result and is covered by tractable case 4 in Theorem 6.8 .

Lemma 6.16. If $A=-1 \wedge E=F= \pm 2 i$, then $C^{2}=-4 \wedge D^{2}=4$, which is tractable.

Proof. Using $w z=A=-1$ and $x y=B=1$, we multiply $\pm 2 i=E=w^{3} x+y z^{3}$ by $w^{3} y$ to get $y^{2} \pm 2 \mathfrak{i} w^{3} y-w^{6}=0$. Similarly, multiplying $\pm 2 \mathfrak{i}=F=w^{3} y+x z^{3}$ by $w^{3} x$ gives $x^{2} \pm 2 \mathfrak{i} w^{3} x-w^{6}=$ 0 . This is the same quadratic polynomial with $x$ and $y$ as indeterminates. Its discriminant is zero; 
thus, $x=y$, which means that $D^{2}=4$. Simplifying (2) yields $C^{2}=-4$ as required. This is covered by tractable case 4 in Theorem 6.8 .

Lemma 6.17. If $A=-1 \wedge E=-F= \pm 2 \mathfrak{i}$, then $C=D=0$, which is tractable.

Proof. Since $0=E+F=C D$, either $C$ or $D$ is zero. Simplifying (2) gives $C^{2}=D^{2}$, so both $C$ and $D$ are zero. This is covered by tractable case 4 in Theorem 6.8.

LEMmA 6.18. If $A=-1 \wedge((E= \pm 2 \mathfrak{i} \wedge F=0) \vee(E=0 \wedge F= \pm 2 \mathfrak{i}))$, then $D^{2}=2 \wedge C^{2}=-2$, which is tractable.

Proof. Since $\pm 2 \mathfrak{i}=E+F=C D$, neither $C$ nor $D$ is zero. Squaring this equation and solving for $C^{2}$ gives $C^{2}=-4 / D^{2}$. In (2), first we substitute for $C^{2}$ to conclude that $D^{2}=2$ and then substitute for $D^{2}$ to conclude that $C^{2}=-2$. This is tractable case 5 in Theorem 6.8.

At this point, every setting of the variables has either been proven tractable over planar graphs or \#P-hard. So far, all of our hardness proofs originate from \#VERTEXCover over 3-regular graphs, which is Holant $\left((0,1,1,1) \mid=_{3}\right)$ (see the proof of Lemma 4.9 in Section 4). Recall that \#VERTEXCOver is \#P-hard even for 3-regular planar graphs [42] and note that all of our gadget constructions are planar, including our interpolation construction in the Group Lemma (see Figure 2(c)). Therefore, all of the \#P-hardness results proved so far still apply when the input is restricted to planar graphs. Thus, what we have achieved is a partition of the entire parameter space $(w, x, y, z)$ into two parts: those that are planar tractable and those that are \#P-hard even restricted to planar graphs.

Within the planar tractable region, there are some cases in which the problem is \#P-hard on general (i.e., nonplanar) graphs, while others are tractable even for general graphs. The region where it is planar tractable but \#P-hard in general is described in Cases 6 and 7 in Theorem 6.8. Case 6 is for symmetric signatures $(x=y)$. We will use the dichotomy already proved for symmetric signatures [35] (see also [36]). Below, we analyze the region where it is planar tractable but \#Phard in general. We will use a lemma from [35] (see also [36]) that can also be found in [34]. The following Lemma 6.20 and Lemma 6.21 prove more than what is logically necessary, but they also illustrate a useful technique reducing the asymmetric case to the symmetric case, that is, a reduction from Lemma 6.20 to Lemma 6.19. Note that they are both planar tractable.

Lemma 6.19 (Lemma 33 of [34]). The problem Holant $\left((w, 1,1, w) \mid==_{3}\right)$ is \#P-hard unless we have that $w \in\{0, \pm 1, \pm i\}$, in which case it is computable in polynomial time.

Lemma 6.20. The problem Holant $\left((w, 1,-1,-w) \mid==_{3}\right)$ is \#P-hard unless we have that $w \in$ $\{0, \pm 1, \pm \mathfrak{i}\}$, in which case it is computable in polynomial time.

Proof. If $w \in\{0, \pm 1, \pm \mathfrak{i}\}$, then the problem is computable in polynomial time (even for nonplanar graphs) by the tractability proof of Theorem 5.1. If $w \notin\{0, \pm 1, \pm \mathfrak{i}\}$, then Gadget 9 and Gadget 10 simulate two symmetric generators. Using REDUCE in Mathematica, we conclude that at least one of the gadgets satisfies the hypothesis for \#P-hardness from Lemma 6.19.

We remark that the use of REDUCE in Mathematica in the proof of Lemma 6.20 is not necessary; the proof is presented as such in order to be succinct. The transition matrices of Gadget 9 and Gadget 10 can be computed as follows. The middle part of both gadgets consists of two copies of $\left(=_{3}\right)$ connected by two parallel copies of $(w, 1,-1,-w)$ in opposite orientations. This gives a binary signature with matrix $\left[\begin{array}{cc}w^{2} & -1 \\ -1 & w^{2}\end{array}\right]$. It follows that the transition matrix of Gadget 9 is

$$
M_{9}=\left[\begin{array}{cc}
w & 1 \\
-1 & -w
\end{array}\right]\left[\begin{array}{cc}
w^{2} & -1 \\
-1 & w^{2}
\end{array}\right]\left[\begin{array}{cc}
w & -1 \\
1 & -w
\end{array}\right]=\left[\begin{array}{cc}
w^{4}+w^{2}-2 w & -2 w^{3}+w^{2}+1 \\
-2 w^{3}+w^{2}+1 & w^{4}+w^{2}-2 w
\end{array}\right] .
$$


Similarly, the transition matrix of Gadget 10 is

$$
M_{10}=\left[\begin{array}{cc}
w & -1 \\
1 & -w
\end{array}\right]\left[\begin{array}{cc}
w^{2} & -1 \\
-1 & w^{2}
\end{array}\right]\left[\begin{array}{cc}
w & 1 \\
-1 & -w
\end{array}\right]=\left[\begin{array}{cc}
w^{4}+w^{2}+2 w & 2 w^{3}+w^{2}+1 \\
2 w^{3}+w^{2}+1 & w^{4}+w^{2}+2 w
\end{array}\right] .
$$

(This can also be obtained by replacing $w$ by $-w$ in Gadget 9 . Note that for $f\left(x_{1}, x_{2}\right)=$ $(w, 1,-1,-w)$, the function $f\left(x_{2}, x_{1}\right)=(w,-1,1,-w)$ becomes its negative after replacing $w$ by $-w$ and that $f$ appears an even number of times in Gadget 9.)

Clearly, we cannot have both entries indexed by $(0,1)$ in $M_{9}$ and $M_{10}$ to be zero. For a nonzero entry, we can normalize it to 1 ; then, we obtain a symmetric function of the form $\left(w^{\prime}, 1,1, w^{\prime}\right)$, as in Lemma 6.19. With this information, it is feasible to verify the claim by direct calculations without the use of REDUCE.

Lemma 6.21. If $w^{3}=\varepsilon z^{3} \wedge x=\varepsilon y$ where $\varepsilon= \pm 1$, then Holant $\left((w, x, y, z) \mid==_{3}\right)$ is \#P-hard unless $x=0 \vee w / x \in\{0, \pm 1, \pm \mathfrak{i}\}$, in which case it is computable in polynomial time.

Proof. If $x=0$, then also $y=0$ and this case, generalized equality, is computable in polynomial time by Theorem 5.1. Now, assume that $x \neq 0$. If $w z \neq 0$, then we apply the holographic transformation $\left[\begin{array}{cc}\alpha & 0 \\ 0 & \alpha^{2}\end{array}\right]$ with $\alpha=\varepsilon z / w$. Note that $\alpha^{3}=\varepsilon z^{3} / w^{3}=1$. The edge signature becomes $(w, x, y, z)\left[\begin{array}{cc}\alpha & 0 \\ 0 & \alpha^{2}\end{array}\right]^{\otimes 2}=\left(\alpha^{2} w, x, y, \alpha z\right)$, while $={ }_{3}$ is unchanged since

$$
\left(\left[\begin{array}{cc}
\alpha & 0 \\
0 & \alpha^{2}
\end{array}\right]^{-1}\right)^{\otimes 3}\left\{\left[\begin{array}{l}
1 \\
0
\end{array}\right)^{\otimes 3}+\left[\begin{array}{l}
0 \\
1
\end{array}\right]^{\otimes 3}\right\}=\left[\begin{array}{c}
\alpha^{2} \\
0
\end{array}\right]^{\otimes 3}+\left[\begin{array}{l}
0 \\
\alpha
\end{array}\right]^{\otimes 3}=\left[\begin{array}{l}
1 \\
0
\end{array}\right]^{\otimes 3}+\left[\begin{array}{l}
0 \\
1
\end{array}\right]^{\otimes 3} .
$$

This reduces to the case $w=\varepsilon z \wedge x=\varepsilon y$. We note that when $w z=0$, this equivalence still holds. We then normalize $x=1$ (since it is nonzero) and replace $z$ with $\varepsilon w$ to obtain the edge signature $(w / x, 1, \varepsilon, \varepsilon w / x)$. Depending on $\varepsilon$, this case is either covered in Lemma 6.19 or Lemma 6.20; thus, we are done.

Proof of Theorem 5.1 (Equivalently Theorem 6.8). We summarize how these lemmas together account for all parameter settings in Theorem 5.1 and, equivalently, Theorem 6.8.

In Section 5, we proved for the tractable cases. Below, we assume that $f=(w, x, y, z)$ is nondegenerate $(A \neq B)$. Cases 6 and 7 in Theorem 6.8, equivalently Case 5 in Theorem 5.1, are proved to be \#P-hard on general 3-regular graphs in Lemma 6.21 (these are tractable on planar graphs). Note, in particular, that Case 7 is equivalent to $C=D=0$. After Lemmas 6.1, 6.2, and 6.3, and by flipping 0 and 1 exchanging $w$ and $z$, or exchanging $x$ and $y$, we may assume that $w x y z \neq 0$ and $|x|=|y|$; thus, $A B \neq 0$. As stated, we then can normalize to $|x|=|y|=1$ and $x=\bar{y}$; thus, $D=x+y \in \mathbb{R}$ with $|D| \leq 2$, and $B=1$, and nondegeneracy means $A \neq 1$. The symmetric case $x=y$ has been proved in [19] conforming to Theorem 5.1; thus, we will assume below that $x \neq y$ and, thus, $|D|<2$. Lemma 6.9 takes care of $A \notin \mathbb{R}$. Below, we can assume that $A \in \mathbb{R}-\{0,1\}$. Lemmas 6.10 and 6.11 take care of when $A \neq-1$ (recall that $C=D=0$ was dealt with earlier.) Below, we may assume that $A=-1$. Lemmas 6.13 and 6.14 handle cases in which $E$ or $F \notin\{0, \pm 2 i\}$. Finally, Lemmas 6.15, 6.16, 6.17 , and 6.18 deal with all remaining cases, where $E, F \in\{0, \pm 2 i\}$. These account for all cases.

\section{ANTI-GADGETS AND PREVIOUS WORK}

To further appreciate the usefulness of anti-gadgets, we show how this technique sheds new light on previous results.

One can find failure conditions for a binary recursive gadget using the following lemma. 


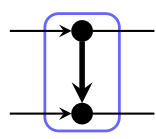

Gadget 11

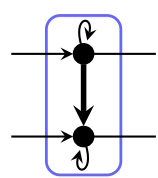

Gadget 12

Fig. 9. Recursive gadgets from [14] on $k$-regular graphs. Bold edges represent parallel edges. In Gadget 11 (resp. Gadget 12), the multiplicity is $k-2$ (resp. $k-4)$ so that the vertices have degree $k$.

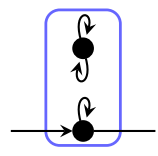

Gadget 13

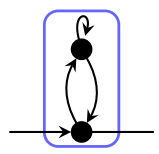

Gadget 14

Fig. 10. Recursive gadgets from [13] on $k$-regular graphs for $k$ even. The gadgets are pictured for $k=4$ but generalize to all even $k \geq 4$ by adding self loops to the vertices.

LEMMA 7.1. Let $G$ be a binary recursive gadget having nonsingular transition matrix $M$. Then, $\left\{M^{i}\right\}_{i \geq 0}$ is a sequence of pairwise linearly independent signatures unless $a_{2}\left|a_{1}\right|^{2}-\left|a_{3}\right|^{2} \overline{a_{2}} a_{0}=0$, where $x^{4}+a_{3} x^{3}+a_{2} x^{2}+a_{1} x+a_{0}$ is the characteristic polynomial of $M$.

Analyzing a failure condition such as $a_{2}\left|a_{1}\right|^{2}-\left|a_{3}\right|^{2} \overline{a_{2}} a_{0}=0$ simultaneously for several gadgets is quite difficult, even with the aid of symbolic computation. Previous work [13, 14] relied heavily on miraculous cancellations in the failure conditions to contend with this. For example, consider the two gadgets in Figure 9. They are from [14], where symmetric (i.e., $x=y)$ signatures $(w, x, y, z)$ were considered on $k$-regular graphs.

After a change of variables $X=w z x^{-2}$ and $Y=(w / x)^{3}+(z / x)^{3}$ and making a few assumptions to guarantee that $M_{11}$ and $M_{12}$ are nonsingular (we omit the details in this discussion), the failure conditions of Gadget 11 and Gadget 12 (when restricted to the real numbers) simplify to

$$
\begin{aligned}
(X-1)^{3}\left(X^{k-2}-1\right)\left(X^{k-2}(X+1)^{2}\left(X^{k-1}+X^{k-2}+X+3 Y+1\right)-Y^{3}\right) & =0, \\
X^{3}(X-1)^{3}\left(X^{k-4}-1\right)\left(X^{k-2}(X+1)^{2}\left(X^{2}+X^{k-2}+X+3 Y+X^{k-3}\right)-Y^{3}\right) & =0 .
\end{aligned}
$$

Assuming that both gadgets fail and $X \notin\{0, \pm 1\}$, this gives two polynomial expressions for $Y^{3}$. Setting these equal to each other and refactoring the expression, we reach a contradiction $X^{k-2}(X+1)^{3}(X-1)\left(X^{k-3}-1\right)=0$, implying that either one or the other gadget works. At the time of this discovery, it was a mystery whether there was any underlying explanation for such miraculous cancellations. Now, we see how anti-gadgets reveal a better understanding of this same gadget pair.

By assuming that $M_{11}$ fails to produce an infinite set of pairwise linearly independent signatures, we have an explicit recursive gadget for $M_{11}^{-1}$. Then, $M_{11}^{-1} M_{12}=\operatorname{diag}(1, X, X, 1)$ clearly produces an infinite set of pairwise linearly independent signatures unless $X$ is zero or a root of unity. Note that in the "gadget language" of $M_{11}^{-1} M_{12}$, the two leading directed edges of Gadget 11 and Gadget 12 simply annihilate each other, as do $k-4$ copies of the vertical edge. The signatures $=_{3}$ at the degree 3 vertices force the matrix $M_{11}^{-1} M_{12}$ to be diagonal. Thus, with almost no effort, we have a strictly stronger result (i.e., over the complex numbers) through the use of an anti-gadget. This also shows that the anti-gadget concept is useful in the symmetric setting as well as the asymmetric setting.

In [13], a similarly fantastic cancellation occurred involving Gadget 13 and Gadget 14 (see Figure 10). They form a suitable gadget and anti-gadget pair, as $M_{13}^{-1} M_{14}$ is a diagonal matrix. While 
this diagonal matrix is not as easy to analyze as the previous example, anti-gadgets would inform the search for such useful gadgets, even if the analysis is carried out with different techniques.

\section{ACKNOWLEDGMENTS}

We thank the anonymous referees for their very careful reading of the article and many helpful suggestions, which greatly helped our presentation. We thank Heng Guo for his many insightful discussions and comments. We also thank him for pointing out an idea similar to that of an antigadget that appeared in the finite characteristic case [31], in which finite order is forced by the characteristic. We thank William Whistler for proofreading parts of this article.

\section{REFERENCES}

[1] Miriam Backens. 2017. A new Holant dichotomy inspired by quantum computation. In 44th International Colloquium on Automata, Languages, and Programming (ICALP'17), July 10-14, 2017, Warsaw, Poland. 16:1-16:14.

[2] Miriam Backens. 2017. A new Holant dichotomy inspired by quantum computation. CoRR abs/1702.00767 (2017).

[3] Rodney J. Baxter. 1982. Exactly Solved Models in Statistical Mechanics. Academic Press London.

[4] Andrei Bulatov and Martin Grohe. 2005. The complexity of partition functions. Theor. Comput. Sci. 348, 2 (2005), $148-186$.

[5] Andrei A. Bulatov. 2013. The complexity of the counting constraint satisfaction problem. F. ACM 60, 5 (2013), 34:1$34: 41$.

[6] Andrei A. Bulatov and Víctor Dalmau. 2007. Towards a dichotomy theorem for the counting constraint satisfaction problem. Inform. and Comput. 205, 5 (2007), 651-678.

[7] Jin-Yi Cai, Pinyan Lu, and Mingji Xia. 2018. Dichotomy for real Holant ${ }^{c}$ problems. In Proceedings of the 29th Annual ACM-SIAM Symposium on Discrete Algorithms (SODA'18), New Orleans, LA, January 7-10, 2018. SIAM, 1802-1821.

[8] Jin-Yi Cai and Xi Chen. 2010. A decidable dichotomy theorem on directed graph homomorphisms with non-negative weights. In FOCS'10. IEEE Computer Society, 437-446.

[9] Jin-Yi Cai, Xi Chen, and Pinyan Lu. 2016. Nonnegative weighted \#CSP: An effective complexity dichotomy. SIAM 7 . Comput. 45, 6 (2016), 2177-2198.

[10] Jin-Yi Cai, Vinay Choudhary, and Pinyan Lu. 2009. On the theory of matchgate computations. Theory Comput. Syst. 45, 1 (2009), 108-132.

[11] Jin-Yi Cai, Heng Guo, and Tyson Williams. 2016. A complete dichotomy rises from the capture of vanishing signatures. SIAM F. Comput. 45, 5 (2016), 1671-1728.

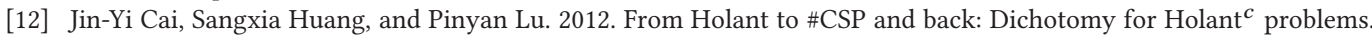
Algorithmica 64, 3 (2012), 511-533.

[13] Jin-Yi Cai and Michael Kowalczyk. 2012. Spin systems on $k$-regular graphs with complex edge functions. Theor. Comput. Sci. 461 (2012), 2-16.

[14] Jin-Yi Cai and Michael Kowalczyk. 2013. Partition functions on $k$-regular graphs with $\{0,1\}$-vertex assignments and real edge functions. Theor. Comput. Sci. 494, 0 (2013), 63-74.

[15] Jin-Yi Cai, Michael Kowalczyk, and Tyson Williams. 2012. Gadgets and anti-gadgets leading to a complexity dichotomy. In Innovations in Theoretical Computer Science 2012, Cambridge, MA, January 8-10, 2012. $452-467$.

[16] Jin-Yi Cai and Pinyan Lu. 2011. Holographic algorithms: From art to science. f. Comput. Syst. Sci. 77, 1 (2011), 41-61.

[17] Jin-Yi Cai, Pinyan Lu, and Mingji Xia. 2009. Holant problems and counting CSP. In STOC'09. ACM, 715-724.

[18] Jin-Yi Cai, Pinyan Lu, and Mingji Xia. 2011. Computational complexity of Holant problems. SIAM f. Comput. 40, 4 (2011), 1101-1132.

[19] Jin-Yi Cai, Pinyan Lu, and Mingji Xia. 2011. A computational proof of complexity of some restricted counting problems. Theor. Comput. Sci. 412, 23 (2011), 2468-2485.

[20] Jin-Yi Cai, Pinyan Lu, and Mingji Xia. 2011. Dichotomy for Holant* problems of Boolean domain. In SODA'11. SIAM, $1714-1728$.

[21] Jin-Yi Cai, Pinyan Lu, and Mingji Xia. 2012. Holographic reduction, interpolation and hardness. Computational Complexity 21, 4 (2012), 573-604.

[22] Jin-Yi Cai, Pinyan Lu, and Mingji Xia. 2014. The complexity of complex weighted Boolean \#CSP. f. Comput. System Sci. 80, 1 (2014), 217-236.

[23] Jin-Yi Cai, Pinyan Lu, and Mingji Xia. 2017. Holographic algorithms with matchgates capture precisely tractable planar \#CSP. SIAM 7. Comput. 46, 3 (2017), 853-889.

[24] Nadia Creignou, Sanjeev Khanna, and Madhu Sudan. 2001. Complexity Classifications of Boolean Constraint Satisfaction Problems. Society for Industrial and Applied Mathematics. 
[25] Martin Dyer, Leslie Ann Goldberg, and Mark Jerrum. 2009. The complexity of weighted Boolean \#CSP. SIAM F. Comput. 38, 5 (2009), 1970-1986.

[26] Martin Dyer, Leslie Ann Goldberg, and Mike Paterson. 2007. On counting homomorphisms to directed acyclic graphs. 7. ACM 54, 6 (2007).

[27] Martin Dyer and Catherine Greenhill. 2000. The complexity of counting graph homomorphisms. Random Struct. Algorithms 17, 3-4 (2000), 260-289.

[28] Martin Dyer and David Richerby. 2010. On the complexity of \#CSP. In STOC'10. ACM, 725-734.

[29] Martin E. Dyer and David Richerby. 2013. An effective dichotomy for the counting constraint satisfaction problem. SIAM f. Comput. 42, 3 (2013), 1245-1274.

[30] Leslie Ann Goldberg, Martin Grohe, Mark Jerrum, and Marc Thurley. 2010. A complexity dichotomy for partition functions with mixed signs. SIAM f. Comput. 39, 7 (2010), 3336-3402.

[31] Heng Guo, Sangxia Huang, Pinyan Lu, and Mingji Xia. 2011. The complexity of weighted Boolean \#CSP modulo $k$. In STACS'11. Schloss Dagstuhl-Leibniz-Zentrum fuer Informatik, 249-260.

[32] Heng Guo, Pinyan Lu, and Leslie G. Valiant. 2013. The complexity of symmetric Boolean parity Holant problems. SIAM f. Comput. 42, 1 (2013), 324-356.

[33] Pavol Hell and Jaroslav Nešetřil. 1990. On the complexity of H-coloring. F. Comb. Theory Ser. B 48, 1 (1990), $92-110$.

[34] Michael Kowalczyk. 2010. Dichotomy Theorems for Holant Problems. Ph.D. Dissertation. University of WisconsinMadison. Retrieved January 17, 2019 from http://cs.nmu.edu/ mkowalcz/research/main.pdf.

[35] Michael Kowalczyk and Jin-Yi Cai. 2010. Holant problems for regular graphs with complex edge functions. In STACS. Schloss Dagstuhl - Leibniz-Zentrum fuer Informatik, 525-536.

[36] Michael Kowalczyk and Jin-Yi Cai. 2016. Holant problems for 3-regular graphs with complex edge functions. Theory Comput. Syst. 59, 1 (2016), 133-158.

[37] László Lovász. 1967. Operations with structures. Acta Math. Hung. 18, 3-4 (1967), 321-328.

[38] H. N. V. Temperley and Michael E. Fisher. 1961. Dimer problem in statistical mechanics-an exact result. Philos. Mag. 6, 68 (1961), 1061-1063.

[39] Salil P. Vadhan. 2001. The complexity of counting in sparse, regular, and planar graphs. SIAM f. Comput. 31, 2 (2001), 398-427.

[40] Leslie G. Valiant. 1979. The complexity of enumeration and reliability problems. SIAM J. Comput. 8, 3 (1979), 410-421.

[41] Leslie G. Valiant. 2008. Holographic algorithms. SIAM J. Comput. 37, 5 (2008), 1565-1594.

[42] Mingji Xia, Peng Zhang, and Wenbo Zhao. 2007. Computational complexity of counting problems on 3-regular planar graphs. Theor. Comput. Sci. 384, 1 (2007), 111-125.

Received February 2018; revised October 2018; accepted November 2018 